\title{
Transcatheter (TAVR) versus surgical (AVR) aortic valve replacement: Occurrence, hazard, risk factors, and consequences of neurologic events in the PARTNER trial
}

D. Craig Miller, MD, ${ }^{a}$ Eugene H. Blackstone, MD, ${ }^{\mathrm{b}}$ Michael J. Mack, MD, ${ }^{\mathrm{c}}$ Lars G. Svensson, MD, PhD, Susheel K. Kodali, MD, ${ }^{\mathrm{d}}$ Samir Kapadia, MD, ${ }^{\mathrm{b}}$ Jeevanantham Rajeswaran, MSc, ${ }^{\mathrm{b}}$ William N. Anderson, PhD, ${ }^{\mathrm{e}}$ Jeffrey W. Moses, MD, ${ }^{\mathrm{d}}$ E. Murat Tuzcu, MD, ${ }^{\mathrm{b}}$ John G. Webb, MD, ${ }^{f}$ Martin B. Leon, MD, ${ }^{\mathrm{d}}$ and Craig R. Smith, MD, ${ }^{\mathrm{d}}$ on behalf of The PARTNER Trial Investigators and Patients, The PARTNER Stroke Substudy Writing Group and Executive Committee

Background: All neurologic events in the PARTNER randomized trial comparing transcatheter aortic valve replacement (TAVR) with surgical aortic valve replacement (AVR) were analyzed.

Methods: High-risk patients with aortic stenosis were stratified into transfemoral $(\mathrm{TF}, \mathrm{n}=461)$ or transapical (TA, $\mathrm{n}=196$ ) strata based on their arterial anatomy and randomized: 657 received treatment assigned ("as treated"), 313 underwent AVR, and 344 TAVR. Neurologic events were prospectively adjudicated by an independent Clinical Events Committee. Multivariable, multiphase hazard analysis elucidated factors associated with increased likelihood of neurologic events.

Results: Forty-nine neurologic events (15 transient ischemic attacks, 34 strokes) occurred in 47 patients (TAVR, $\mathrm{n}=31$; AVR, $\mathrm{n}=16$ ). An early peaking high hazard phase occurred within the first week, which declined to a constant late hazard phase out to 2 years. The risk in the early phase was higher after TAVR than AVR, and in the TAVR arm in patients with a smaller aortic valve area index. In the late risk phase, the likelihood of neurologic event was linked to patient-related factors in both arms ("non-TF candidate," history of recent stroke or transient ischemic attack, and advanced functional disability), but not by treatment (TAVR vs AVR) or any intraprocedural variables. The likelihood of sustaining a neurologic event was lowest in the AVR subgroup in the TF stratum during all available follow-up.

Conclusions: After either treatment, there were 2 distinct hazard phases for neurologic events that were driven by different risk factors. Neurologic complications occurred more frequently after TAVR than AVR early, but thereafter the risk was influenced by patient- and disease-related factors. (J Thorac Cardiovasc Surg 2012;143:832-43)

Earn CME credits at

http://cme.ctsnetjournals.org
Supplemental material is available online.

In the randomized PARTNER (Placement of AoRTic TraNscathetER Valves) trial ${ }^{1}$ (ClinicalTrials.gov identifier NCT00530894), neurologic events occurred more frequently after transcatheter aortic valve replacement (TAVR) than after surgical aortic valve replacement (AVR) at 30 days and 1 year in high surgical risk patients with severe aortic stenosis (AS). ${ }^{2}$ These events were associated with substantial mortality ${ }^{1,2}$ and have become a focal point in evaluating the safety of TAVR.

The aim of this study was to investigate comprehensively the nature of all neurologic events occurring after TAVR or AVR, the temporal pattern of their occurrence and association with risk factors, the likelihood of such an event occurring within the lifetime of patients, and the mortality consequences of these events. Such knowledge will be useful in identifying the cause of these events, devising ways to 


$\begin{array}{ll}\text { Abbreviations and Acronyms } \\ \text { AF } & =\text { atrial fibrillation } \\ \text { AS } & =\text { aortic stenosis } \\ \text { AT } & =\text { as treated } \\ \text { AVAI } & =\text { aortic valve area index } \\ \text { AVR } & =\text { aortic valve replacement } \\ \text { BAV } & =\text { balloon aortic valvuloplasty } \\ \text { CABG } & =\text { coronary artery bypass grafting } \\ \text { CEC } & =\text { Clinical Event Committee } \\ \text { ITT } & =\text { intent to treat } \\ \text { PARTNER } & =\text { Placement of AoRTic } \\ & \text { TraNscathetER Valves (trial) } \\ \text { STS } & =\text { The Society of Thoracic Surgeons } \\ \text { TA } & =\text { transapical } \\ \text { TAVR } & =\text { transcatheter aortic valve } \\ \text { TF } & \text { replacement } \\ \text { TIA } & =\text { transfemoral } \\ & =\end{array}$

prevent them, and providing decision support and realistic expectations for patients.

\section{PATIENTS AND METHODS}

The PARTNER trial design, inclusion and exclusion criteria, and other details have been previously reported ${ }^{1,2}$ and are summarized in Appendix E1.

\section{Patients}

Among 699 intent to treat patients (ITT) with severe AS who were high risk $\left(\sim 10 \%-15 \%\right.$ Society of Thoracic Surgeons [STS] mortality score $\left.{ }^{3,4}\right)$ for open AVR, ${ }^{2} 657$ who received treatment assigned ("as treated," or AT) were stratified into either the transfemoral $(\mathrm{TF}, \mathrm{n}=461)$ or transapical (TA, $\mathrm{n}=196$ ) stratum (see CONSORT diagram, Figure E1). Patients were classified as either TF or TA based on a "TF first" philosophy according to whether their arterial anatomy was suitable ( $>7-\mathrm{mm}$ lumen diameter) for the 22Fr or 24Fr sheaths required for insertion of TF 23-mm and 26-mm SAPIEN valves (Edwards Lifesciences, Irvine, Calif). They were then randomized within each stratum to undergo either TAVR $(n=344)$ or AVR $(\mathrm{n}=313$, Figure E1), thereby creating separate parallel control (AVR) subgroups for direct comparison with TAVR patients within each stratum. Among the 461 AT patients in the TF stratum, 240 were randomized to TAVR and 221 to AVR. Beginning April 6, 2008, about 1 year after the TF limb of the trial started, if a patient anatomically was not a suitable candidate for TF-TAVR, TA-TAVR was performed through a small intercostal incision over the left ventricular apex. There were 196 AT patients in the TA stratum: 104 randomized to TAVR, and 92 to AVR.

Pertinent characteristics of the 657 PARTNER high-risk AT patients according to assigned stratum (TA or TF) are summarized in Table E1. They had critical AS (mean aortic gradient $=41 \pm 13$ vs $44 \pm 15 \mathrm{~mm} \mathrm{Hg}[ \pm 1$ $\mathrm{SD}$ ], aortic valve area $=0.7 \pm 0.2$ vs $0.6 \pm 0.2 \mathrm{~cm}^{2}$ ), were elderly (average age $83 \pm 6$ vs $84 \pm 7$ years, respectively), very high risk (STS score $=11.9 \% \pm 3.6 \%$ vs $11.7 \% \pm 3.3 \%, \log$ EuroSCORE $=30 \% \pm$ $16 \%$ vs $29 \% \pm 16 \%$ ), highly symptomatic (New York Heart Association class III-IV in $94 \%$ vs $95 \%$ ), and had many serious medical comorbidities. Significantly more patients with previous carotid endarterectomy or stenting, peripheral arterial bypass grafting/stenting/balloon dilatation, coronary artery bypass grafting $(\mathrm{CABG})$, cerebrovascular disease, and peripheral arterial vascular disease were in the TA stratum compared with the TF stratum, reflecting more generalized arteriosclerotic burden (Figure E2). This was confirmed in a parsimonious multivariable logistic regression model (see Appendix E1 methods and Table E2).

The pooled patient characteristics were similar according to whether they were randomized to TAVR or AVR (ie, both TF and TA strata combined, Table E3). Table E4 lists important clinical features for all 4 subgroups.

\section{Patient Management}

Patients received heparin during the TAVR procedure, and dual antiplatelet therapy (aspirin and clopidogrel) was recommended for 6 months postoperatively. Warfarin anticoagulation and the duration of antiplatelet therapy were left to the discretion of local physicians.

\section{End Points}

End points included were all neurologic events and all-cause mortality for all available follow-up. All adverse clinical events were independently reviewed in a blinded, prospective manner by the Clinical Event Committee (CEC), including neurologic complications. See Appendix E1 for complete details concerning neurologic assessment. Our ability to determine precisely the severity and permanence of neurologic damage was limited in the PARTNER-I trial. All neurologic events were combined to provide sufficient power for a hazard function model and to analyze the clinical importance of all types of neurologic complications. ${ }^{5,6}$

\section{Data Analysis}

See Appendix E1 for detailed statistical methods. In brief, the instantaneous temporal pattern of risk of neurologic events was characterized by multiphase hazard function methodology that distinguished early from later phases of risk. Risk factors for neurologic events were identified for each hazard phase, including detailed search for interaction terms. Likelihood of a neurologic event occurring during a patient's lifetime was estimated by competing risks analysis, with death as the competing risk. Mortality consequences of a neurologic event were estimated by analysis of death after such an event compared with expected deaths had a neurologic event not occurred.

\section{RESULTS}

\section{Nature of Neurologic Events}

Forty-nine neurologic events (15 transient ischemic attacks [TIAs], 34 strokes) occurred in 47 patients (TAVR: 31 patients, 31 events; AVR: 16 patients, 18 events). Timing and type of neurologic events are shown in Table 1. Fiftyone percent $(24 / 49)$ were procedure related $(<10$ days $)$ and $38 \%(18 / 49)$ occurred within the first 2 days. Eighteen $(58 \%)$ of 31 TAVR patients who sustained neurologic events had a major stroke, similar to $69 \%(11 / 16)$ of the AVR patients. At 10 days, the major stroke rate was 3.4\% $(12 / 344)$ in the TAVR and $1.9 \%(6 / 313)$ in the AVR treatment arms. The neurologic event was a TIA in $26 \%$ (12/ 47) of patients ( $29 \%$ of the total 49 events). Etiology was judged to be ischemic in $72 \%$, ischemic evolving to hemorrhagic in 4\%, unknown in $24 \%$, and hemorrhagic in none. One AVR patient who sustained a major stroke at 7 days had a second event (TIA) at 9 days; another AVR patient who had a TIA between 1 month and 1 year experienced a second TIA 411 days postoperatively. Twenty ( $43 \%$ ) of the $47 \mathrm{pa}-$ tients with a neurologic event ultimately died, compared with $29 \%(174 / 610)$ of those without a neurologic event. 
TABLE 1. Timing and type of 49 neurologic events in 47 patients

\begin{tabular}{|c|c|c|c|c|c|c|c|c|}
\hline AT time & $0-2 d$ & 3-5 d & $6-10 \mathrm{~d}$ & $11-30 \mathrm{~d}$ & $31-364 \mathrm{~d}$ & $1-2 y$ & $2-3 y$ & Total \\
\hline & \multicolumn{8}{|c|}{ TAVR } \\
\hline TIA & 2 & 0 & 0 & 1 & 4 & 1 & 0 & 8 \\
\hline Minor CVA & 3 & 0 & 0 & 0 & 0 & 2 & 0 & 5 \\
\hline Major CVA & 7 & 2 & 3 & 0 & 5 & 1 & 0 & 18 \\
\hline \multirow[t]{2}{*}{ Subtotal } & 12 & 2 & 3 & 1 & 9 & 4 & 0 & 31 \\
\hline & \multicolumn{8}{|c|}{ AVR } \\
\hline TIA & 0 & 0 & $0(1 *)$ & 0 & 3 & $1\left(1^{*}\right)$ & 0 & $4(2 *)$ \\
\hline Minor CVA & 1 & 0 & 0 & 0 & 0 & 0 & 0 & 1 \\
\hline Major CVA & 5 & 0 & 1 & 1 & 1 & 2 & 1 & 11 \\
\hline Subtotal & 6 & 0 & $1\left(1^{*}\right)$ & 1 & 4 & $3\left(1^{*}\right)$ & 1 & $16(2 *)$ \\
\hline Total & & & & & & & & $47\left(49^{*}\right)$ \\
\hline
\end{tabular}

\section{Temporal Pattern and Risk Factors}

The instantaneous risk of neurologic events after TAVR or AVR demonstrated an early, peaking high hazard phase in the first postoperative week followed by a constant lower hazard phase throughout the duration of follow-up (Figure 1,A). This temporal pattern is similar to that seen for stroke after CABG, where the peak hazard occurs about postoperative day $2{ }^{7}$ The shape of the hazard function suggested that different risk factors were present early after either procedure compared with later (nonproportional hazards), likely representing different mechanisms; Table 2 summarizes the early and late independent predictors of neurologic events. In the early high hazard phase, TAVR (Figure 1, A) and smaller aortic valve area index (AVAI, $\mathrm{cm}^{2} / \mathrm{m}^{2}$ ) in the TAVR arm (Figure $1, B$ ) were associated with a significantly increased risk of a neurologic event; in the AVR treatment arm the effect of smaller AVAI was not observed. History of cerebrovascular disease was possibly associated with higher risk. The early high hazard phase for patients assigned to the TF stratum (Figure 1, $C$ ) underscored the higher risk of neurologic events in the TF-TAVR arm than in the AVR subgroup. For patients in the TA stratum, early hazard after TA-TAVR in the first week (Figure 1, $D$ ) was slightly lower than after AVR but more prolonged out to about 2 weeks, then slowly rose to 24 months in the late hazard phase (Figure 1,E).

These hazard functions translated into an early separation of neurologic event curves during the first week between the pooled TAVR and AVR treatment arms (Figure 2, A), which thereafter slowly rose in parallel. At 2 years, the point estimate of neurologic events was $6.7 \%$ in the pooled AVR arm and $11 \%$ in the pooled TAVR arm. For patients in the TF stratum, the point estimate of neurologic events was $2.4 \%$ after AVR versus $6 \%$ after TF-TAVR at 1 year and $3.4 \%$ and $7.4 \%$, respectively, at 2 years (Figure 2,B). For patients in the TA stratum, Figure 2, $C$, illustrates that occurrence of stroke or TIA was similar in both treatment arms but higher than that observed in the TF stratum: $10 \%$ after AVR versus $12 \%$ after TA-TAVR at 1 year.

During the late hazard phase, treatment arm was not a significant risk factor for neurologic events. Rather, patientrelated and disease-related characteristics were incremental risk factors for neurologic events (see Table 2), including advanced functional impairment (New York Heart Association functional class) (Figure 3, A) and history of stroke within 6 to 12 months. The point estimate of new neurologic event in those with a previous stroke or TIA was $23 \%$ at 2 years compared with $8.9 \%$ for those without a neurologic event (Figure 3, B). (NB: Stroke or TIA within 6 months was a PARTNER exclusion criterion.) Patients with a history of percutaneous coronary intervention or chronic obstructive pulmonary disease had lower late risk. The strongest and most statistically significant late risk factor was being judged to be a "non-TF candidate," viz, assigned to the TA stratum. Importantly, the strength of this "non-TF candidate" factor in the TAVR treatment arm $(12 \%$ of TATAVR patients had sustained a neurologic event by 1 year vs $6 \%$ in the TF-TAVR subset, Figure 3, $C$ ) was just as powerful in the AVR arm (10\% of AVR patients in the TA stratum had sustained a neurologic event by 1 year vs $2.4 \%$ of AVR patients in the TF stratum, Figure 3,D). Hence, the adverse effect of TA stratum affected both treatment arms similarly.

Presence of arteriosclerotic peripheral arterial disease was not identified as a risk factor. Two variables representing preprocedural cardiac arrhythmias were assessed (one being clinical history of arrhythmias that was loosely considered to represent atrial fibrillation [AF], and the other an adjudicated AF variable determined on a single electrocardiogram by the ECG Core Lab). Both were examined in this analysis, but neither was identified to be a risk factor for neurologic events in either hazard phase.

Only 29 major strokes occurred, precluding robust statistical analysis. The confidence limits for major stroke in the pooled (TA and TF strata combined) TAVR and AVR patients overlapped (Figure E3, A): point estimates at 2 years 


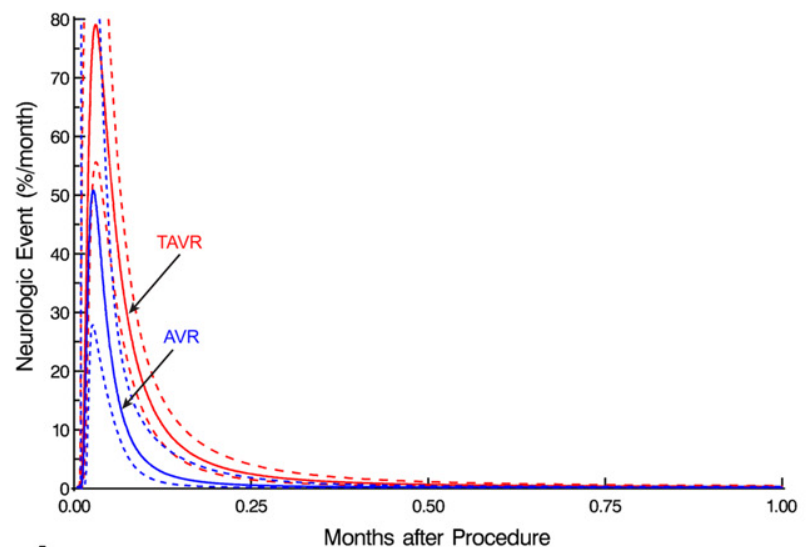

A

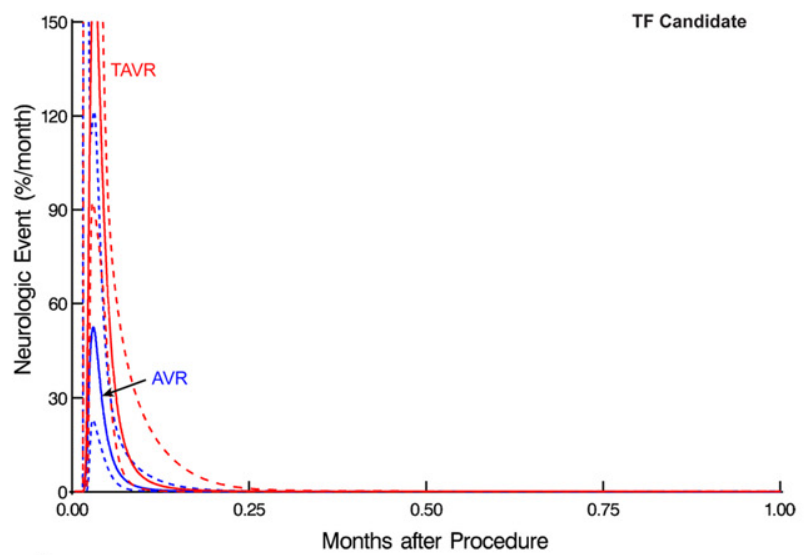

C

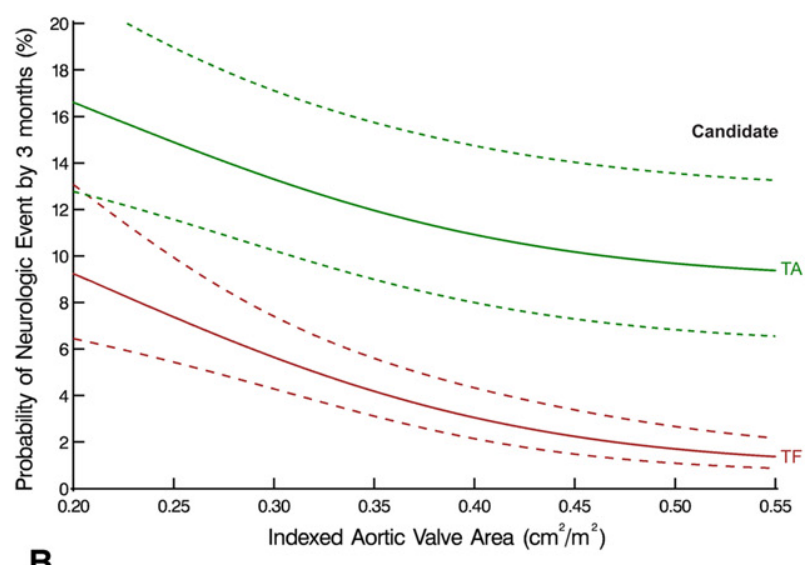

B

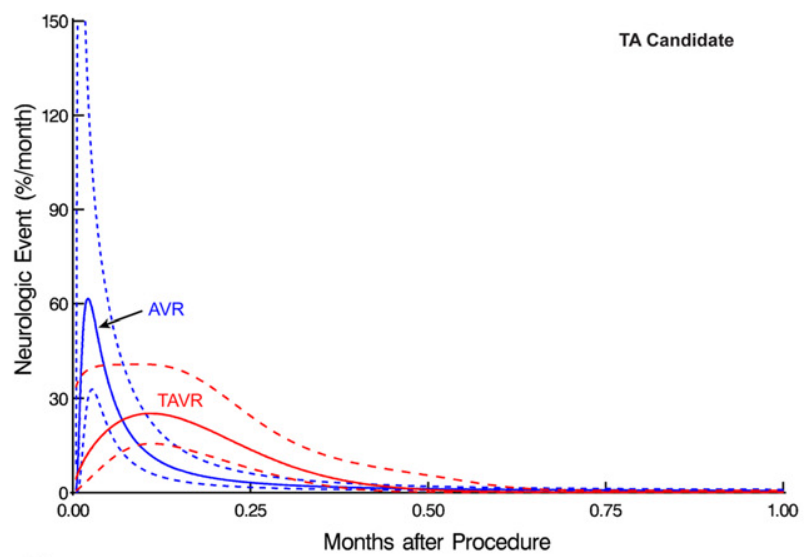

D

TA Candidate

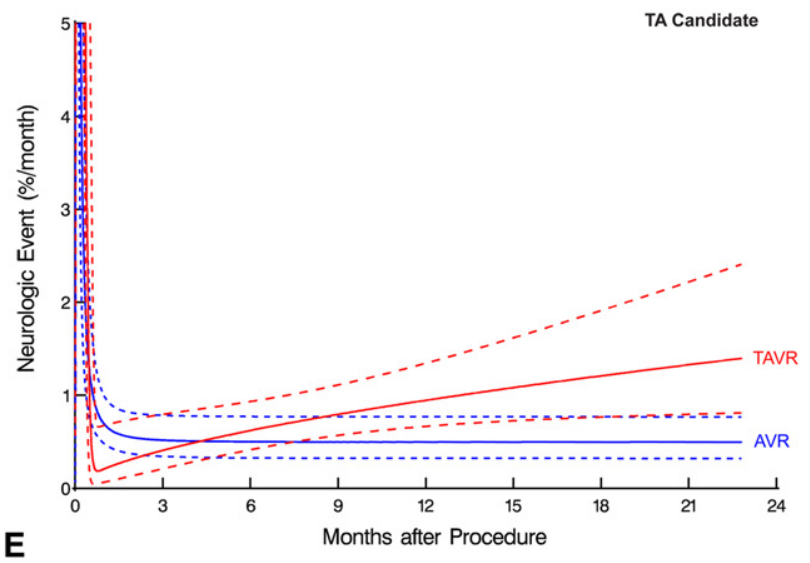

FIGURE 1. Risk of neurologic events after transcatheter aortic valve replacement (TAVR) and surgical aortic valve replacement (AVR). A, Instantaneous hazard (calculated as percent/month) of a neurologic event after procedure for pooled TAVR and AVR arms. Solid lines represent parametric estimates and are enclosed within asymmetric confidence bands equivalent to \pm 1 standard error. Red curve is for TAVR and blue curve for AVR. B, Probability of neurologic event at 3 months within the TAVR treatment arm as a function of native aortic valve area index (AVAI) and assignment to the transfemoral (TF) or transapical (TA) stratum. This depiction is a nomogram of the equation represented in Table 2 with values for all variables held constant except for whether or not the patient was a suitable candidate for TF-TAVR. The nomogram was solved with the following values for variables in the model: TAVR $=$ yes, cerebrovascular disease $=$ no, New York Heart Association $=$ IV, stroke within 6 to 12 months $=$ no, history of percutaneous coronary intervention $=$ no, chronic obstructive pulmonary disease $=$ no. Solid lines represent parametric estimates and are enclosed within asymmetric confidence bands equivalent to \pm 1 standard error. C, Hazard function for neurologic events after either TAVR or AVR among patients assigned to the transfemoral (TF) stratum. Depiction is as in Figure 1, A. D, Hazard function for neurologic events after either TAVR or AVR among patients assigned to the transapical (TA) stratum. Depiction is as in Figure 1, A. E, Instantaneous hazard to 24 months for neurologic events after either TAVR or AVR among patients assigned to the transapical (TA) stratum. Depiction is as in Figure 1, A. 
TABLE 2. Incremental risk factors for neurologic events after procedure (including factors specific to procedure performed)

\begin{tabular}{lccc}
\hline \multicolumn{1}{c}{ Risk factor } & Coefficient \pm SD & $\boldsymbol{P}$ & R $(\%)$ \\
\hline Early hazard phase & & & \\
TAVR* & $2.21 \pm 0.68$ & .001 & 59 \\
Cerebrovascular disease & $0.76 \pm 0.45$ & .09 & 44 \\
(Smaller) indexed native & $-11.8 \pm 5.1$ & .02 & 57 \\
$\quad$ aortic valve areat in & & & \\
TAVR group & & & \\
Constant hazard phase & & & \\
TAVR & $0.40 \pm 0.43$ & .4 & 22 \\
(Higher) NYHA & $0.95 \pm 0.40$ & .02 & 75 \\
Stroke within 6-12 mo & $1.93 \pm 0.64$ & .002 & 60 \\
Non-TF-TAVR candidate & $2.3 \pm 0.45$ & $<.0001$ & 96 \\
History of PCI (less risk) & $-1.60 \pm 0.63$ & .01 & 77 \\
COPD (less risk) & $-1.06 \pm 0.47$ & .03 & 79 \\
\hline
\end{tabular}

$\mathrm{R}(\%)$, or bagging reliability, is interpreted as the probability of $P<.07$ and represents the proportion of 500 bootstrap analyses in which this variable was retained with $P<.07 . S D$, Standard deviation; TAVR, transcatheter aortic valve replacement; NYHA, New York Heart Association; TF, transfemoral; $P C I$, percutaneous coronary intervention; COPD, chronic obstructive pulmonary disease. *Once interactions are considered, the variable TAVR becomes an offset to the intercept term in the model. $\dagger(\text { Valve area/body surface area })^{2}$ squared transformation.

were $6.1 \%$ in the TAVR treatment arm versus $4.5 \%$ in the AVR treatment arm. There was, however, both an early (Figure E3, B) and a continuing late risk of major stroke (Figure E3, $C$ ). After AVR the early phase estimates for major stroke and all neurologic events were similar. Although the area under the major stroke early hazard phase curve for TAVR was insignificantly less than after AVR $(P=.3)$, the duration of the early phase extended to about 1 week compared with the initial 1 to 2 days after AVR (Figure E3, B).

\section{Likelihood of Neurologic Event-Free Survival}

The likelihood of being alive and free of a neurologic event is shown according to treatment arm (TAVR vs AVR) and TF or TA stratum in Figure E4, $A$ to $D$, superimposed with the likelihood of experiencing a neurologic event or of dying before such an event occurs. Values for these competing risks of neurologic event and death from the 4 subsets are stacked in a single graph (Figure 4) with an expanded vertical scale to illustrate the "mortality-adjusted" likelihood of being alive and not having sustained a neurologic event. Likelihood at 2 years was lowest $(2.6 \%$, most favorable outcome) for the AVR patients in the TF stratum versus $6.9 \%$ for the TF-TAVR patients. For either TAVR or AVR patients in the TA stratum, the likelihood was substantially higher, but similar between treatment arms; the point estimate at 1 year was $9.1 \%$ for AVR patients and $11 \%$ for TAVR patients. Note that the curves representing the TA stratum patients (treated either way) rose steeply late postoperatively compared with the corresponding flat curves in the TF stratum, consistent with the "non-TF candidate" variable being the dominant predictor of stroke or TIA in the late hazard phase (Table 2).

\section{Mortality Consequences}

Observed mortality after a neurologic event was higher than that expected had a neurologic event not occurred (based on the competing risk estimates for death before a neurologic event). Thus, as explained in Appendix E1, conditional survival, which starts at $100 \%$ at the time of a neurologic event, was computed on the basis of the survival experience of all patients, thereby allowing meaningful comparison of prognoses after an event. This is shown in Figure E5, $A$ to $F$, for the pooled AVR, TF-TAVR, and TA-TAVR groups; for clarity, estimated life expectancy was expressed as the hazard ratio of observed to expected mortality (Figure 5). For the pooled AVR patients, the hazard ratio was high early after the neurologic event for about 6 weeks; thereafter, it returned to 1.0 (expected mortality risk) out to 1 year (see Figure 5, A). For TAVR-TF patients, the high risk of mortality after an event remained elevated over the first year, only slowly approaching 1.0 (Figure 5, $B$ ). For TAVR-TA patients, the elevated risk of death after a neurologic event remained high throughout the first year (Figure 5, C).

\section{DISCUSSION}

Although surgical AVR remains the gold standard, at least one third of symptomatic patients with AS may not undergo AVR owing to comorbidities such as advanced age and frailty, life-limiting other medical diseases, or patient choice. ${ }^{8}$ TAVR outside the United States is an alternative to AVR and in 2010 accounted for $24 \%(3629 / 15,318)$ of all AVRs performed in 79 centers in Germany (1450 via a transvascular approach and 2179 via the TA approach). ${ }^{9}$ Observational studies have demonstrated the utility of TAVR using either the balloon-expandable SAPIEN bovine pericardial or the self-expanding CoreValve (Medtronic, Inc, Minneapolis, Minn) porcine pericardial device in high-risk and inoperable patients ${ }^{10,11}$; while procedural success has become the norm, complications (including stroke and vascular complications) have been noteworthy. The risk of stroke after TAVR has been reported in the $2.4 \%$ to $9.1 \%$ range for TF-TAVR and $1.5 \%$ to $6.7 \%$ for TA-TAVR. ${ }^{10,11}$ This variability may be explained by differences in study design (retrospective vs prospective $e^{5,6}$ ), sample size, methodology used, ${ }^{5}$ patient substrate and site-specific factors, and voluntary self-reporting. ${ }^{5}$ As shown in many different stroke studies, lack of independent adjudication leads to underreporting, making it impossible to compare devices or approaches.

The PARTNER trial was the first prospective randomized investigation comparing TAVR and AVR in inoperable and high-risk operable patients with severe AS. ${ }^{1,2}$ Inoperable (cohort B) patients were randomized between TAVR and optimal medical therapy, which demonstrated an absolute $20 \%$ reduction in mortality at 1 year after TAVR, along 

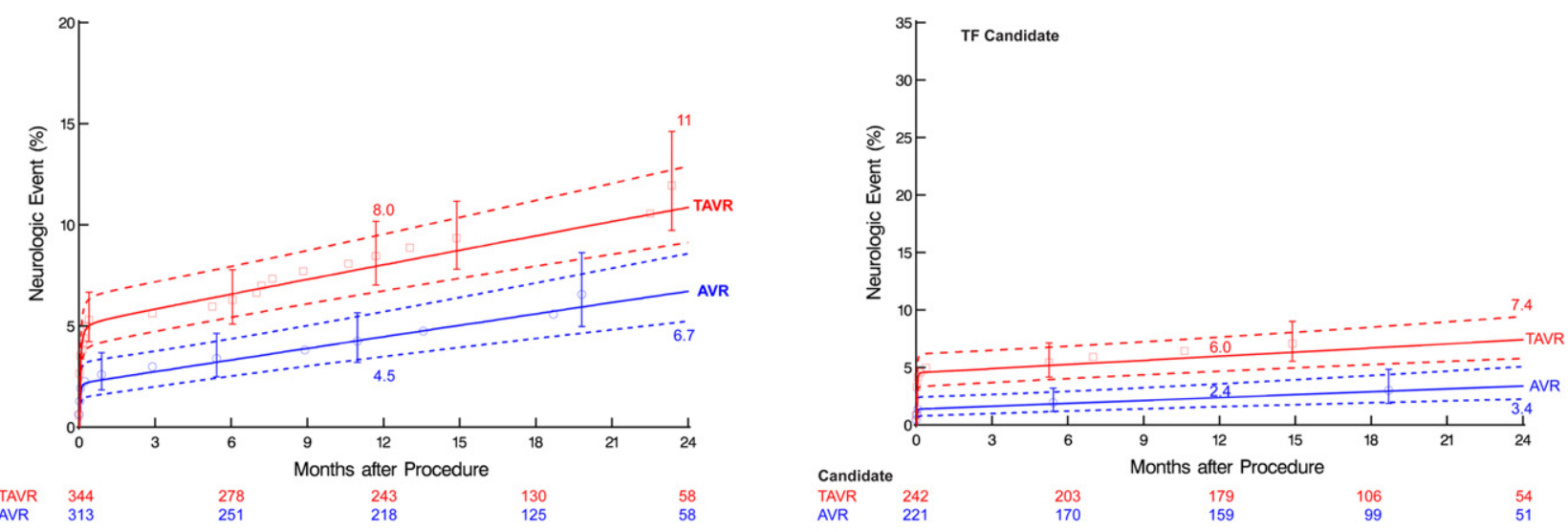

A

B

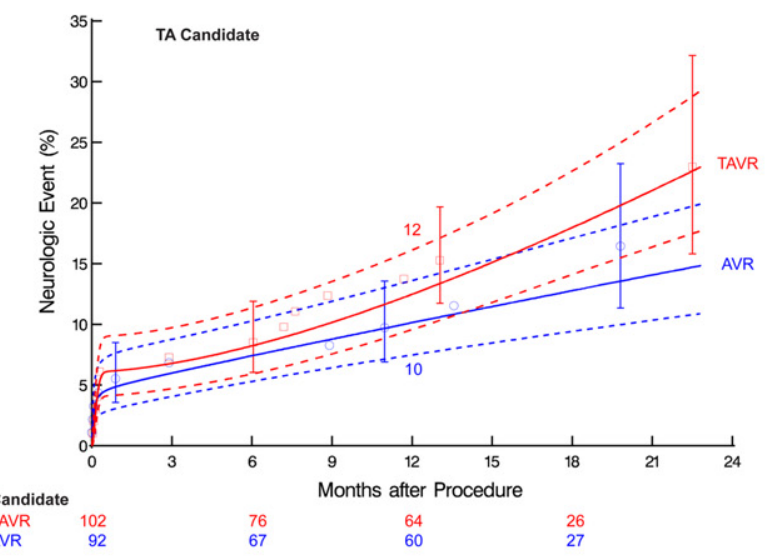

C

FIGURE 2. Occurrence of neurologic events after transcatheter aortic valve replacement (TAVR) and surgical aortic valve replacement (AVR). Each symbol represents an event; vertical bars are confidence limits equivalent to \pm 1 standard error, and solid lines represent parametric estimates enclosed within dashed confidence bands indicating \pm 1 standard error. Number of patients remaining at risk is denoted below the horizontal axis at 6-month intervals. Red curve and open squares depict the TAVR treatment arm, and blue curve and open circles the surgical AVR arm. Numbers within the plot are point estimates at 12 and 24 months. A, Occurrence of neurologic events according to treatment arm. B, Occurrence of neurologic events among patients assigned to the transfemoral $(T F)$ stratum. C, Occurrence of neurologic events among patients assigned to the transapical (TA) stratum.

with reduced symptoms. ${ }^{1}$ Procedural complications occurred, however, including a $6.7 \%$ incidence of neurologic events at 30 days with a $5 \%$ major stroke rate. ${ }^{1}$ This higher risk of neurologic complications compared with medical therapy is acceptable in these inoperable patients given the marked symptomatic and survival benefit.

Stroke also is a widely recognized complication after surgical AVR, especially in elderly patients, ${ }^{3}$ but unfortunately in the literature stroke is not strictly defined, neurologic events are not independently adjudicated, and the majority of the reports are from voluntary databases or single-center experiences. ${ }^{12,13}$ Prospective, more rigorous reports are rare. ${ }^{5}$ Voluntary self-reported databases, including the STS database, ${ }^{3,4}$ underestimate the risk of stroke, use varying subjective definitions, and lack independent adjudication of adverse events or universal auditing. Stroke rates in elderly, high-risk patients undergoing surgical AVR generally ranged from $2 \%$ to $4 \%^{3-5}$; however, in 2 recent single- center reports of high-risk patients with previous CABG undergoing surgical AVR, the stroke rates were $11 \%$ for those older than 75 years ${ }^{12}$ and $8 \%$ in those over $80 .{ }^{13}$

High-risk, operable patients (PARTNER cohort A) were randomized between TAVR and AVR $($ ITT, $n=699){ }^{2} \mathrm{Neu}-$ rologic events were more frequent in TAVR patients. The incidence of neurologic events in the TAVR arm was twice as high as in the AVR arm $(5.5 \%$ vs $2.4 \%$ at 30 days $[P=.04]$, and $8.3 \%$ vs $4.3 \%$ at 1 year $[P=.04]$, respectively, ITT cohorts), although the rate of major stroke was not significantly different $(3.8 \%$ vs $2.1 \%[P=.20]$ at 30 days and $5.1 \%$ vs $2.4 \%[P=.07]$ at 1 year, respectively). ${ }^{2}$ For the PARTNER high-risk AT patients, the rate of neurologic events in the TF stratum was 3-fold higher after TAVR $(\mathrm{n}=240)$ than after AVR $(\mathrm{n}=221): 4.6 \%$ vs $1.4 \%$ at 30 days $(P=.04)$ and $6.1 \%$ vs $1.9 \%$ at 1 year $(P=.03)$, (Supplementary Appendix Table 9, see Smith and associates ${ }^{2}$ ), which attracted scrutiny. ${ }^{14}$ This low 30-day neurologic 


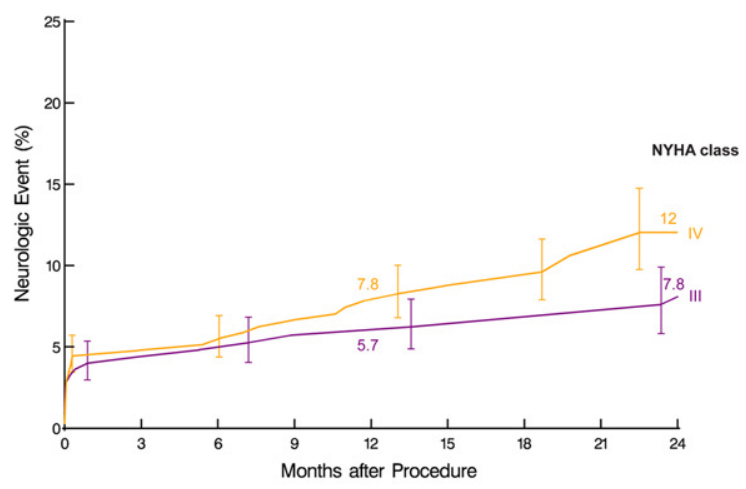

A

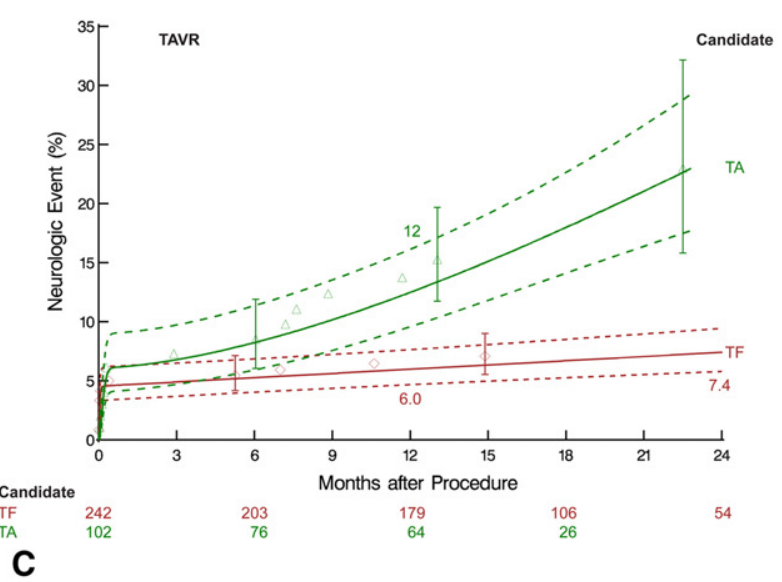
$\begin{array}{ll}\text { Candidate } \\ \text { TF } & 242 \\ \text { TA } & 102 \\ \text { C } & \end{array}$
203
231 120
119

63

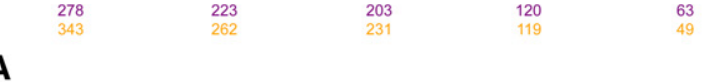

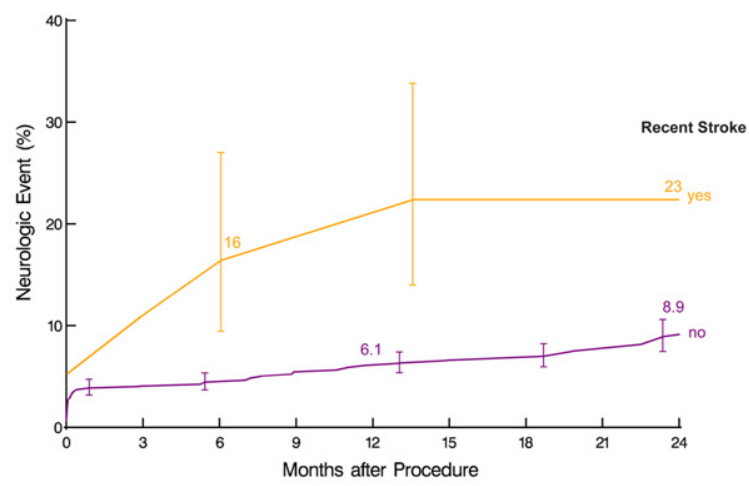

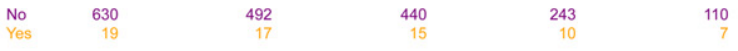

B

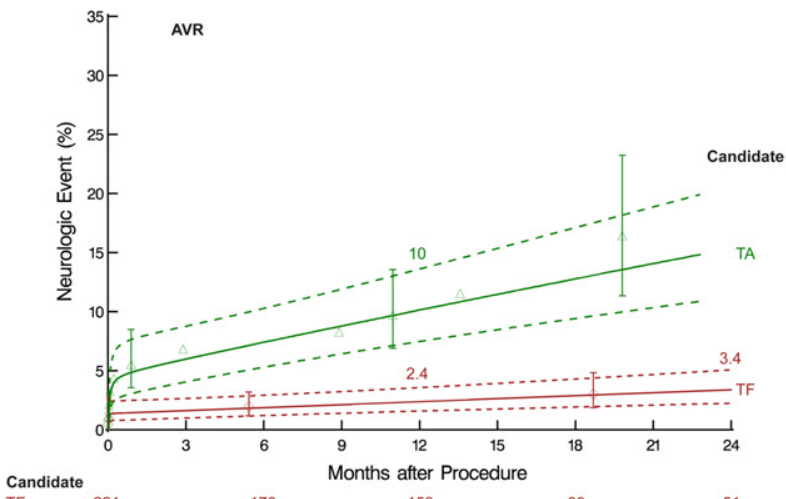

$\begin{array}{lr}\text { Candidate } & \\ \text { TF } & 221 \\ \text { TA } & 92 \\ \text { D } & \end{array}$
159
60

99
27

FIGURE 3. Occurrence of neurologic events for all patients (transcatheter aortic valve replacement [TAVR] and surgical aortic valve replacement [AVR] treatment arms combined, except as noted) stratified by risk factors (see Table 2). Vertical bars are confidence limits equivalent to \pm 1 standard error. Number of patients at risk is denoted below the horizontal axis at 6-month intervals. Numbers within the plot are point estimates at 12 and 24 months. A, Actuarial estimates of neurologic events stratified by New York Heart Association (NYHA) functional class. B, Actuarial estimates of neurologic events stratified by presence or absence of stroke or transient ischemic attack in last 6 to 12 months. C, Occurrence of neurologic events after TAVR, stratified by whether the patient was assigned to transfemoral (TF) or transapical (TA) stratum (this stratum designator was applied to patients in both treatment arms [TAVR or AVR]). Depiction is as in Figure 2, A, except that the brown curve is for the TF candidates who underwent TAVR and the green curve for TA-TAVR patients. $\mathrm{D}$, Occurrence of neurologic events after AVR stratified by whether the patient was assigned to the transfemoral (TF) or transapical (TA) stratum (this stratum designator applied to patients in both treatment arms [TAVR or AVR]). Depiction is as in Figure 3, $C$.

event rate of $1.4 \%$ after AVR is striking given how old and sick the PARTNER cohort A patients were, rivaling the $1.3 \%$ stroke rate in 2689 younger AVR patients. ${ }^{15}$ In the TA stratum, the neurologic event rates were higher in both the TAVR and AVR treatment arms $(7.9 \%$ vs $5.5 \%$ at 30 days, $P=.5 ; 14.1 \%$ vs $9.7 \%$ at 1 year, $P=.37$, respectively) and of similar magnitude (Supplementary Appendix Table 11, see Smith and associates ${ }^{2}$ ). Similar to the ITT analyses, the incidence of major stroke in the AT patients was similar between the TAVR and AVR arms in both strata. ${ }^{2}$ If a neurologic event occurred, however, the ensuing 1-year mortality rate was higher after TAVR than after $\mathrm{AVR}^{2}$; however, the composite end point of death and major stroke was not significantly different between the TAVR and AVR patients at 1 year $(26.5 \%$ vs $28 \%$, respectively $[P=.7])$.
In contrast to the earlier PARTNER trial results, which were truncated at 1 year, ${ }^{1,2}$ this analysis looked at all neurologic events regardless of time and revealed several novel findings: (1) In both treatment arms, there was an early peaking high hazard phase of neurologic events within the first week, which was followed thereafter by a late constant hazard phase. (2) Neurologic events of all types continued unabated, at a lower level, throughout follow-up. (3) The temporal pattern of neurologic events was generally similar after AVR and TAVR, but the early risk of neurologic events was substantially higher after TAVR, particularly in the TF stratum (Figure 1, A). (4) Within the TAVR treatment arm, the early risk of neurologic event was elevated in those with a smaller AVAI (Figure 1, $B)$, most likely owing to the tighter valves having more calcification that potentially could embolize during TAVR. (5) 


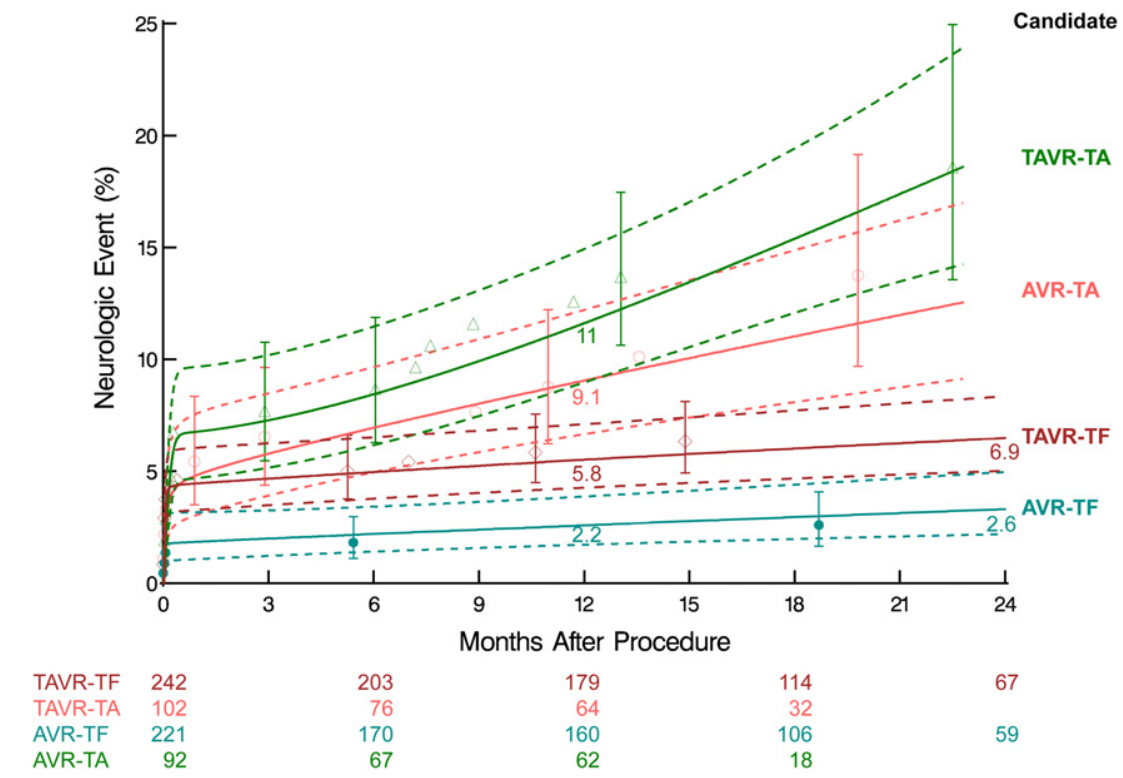

FIGURE 4. Likelihood of neurologic event when the competing risk of death is taken into consideration in each of the 4 treatment subgroups. Depicted in light blue is the curve for the likelihood of a neurologic event after aortic valve replacement $(A V R)$ in the TF stratum, the salmon-colored curve is that for AVR patients assigned to the TA stratum, the brown curve is for the transfemoral transcatheter aortic valve replacement $(T F-T A V R)$ patients in the TF stratum, and the green curve is for the TA-TAVR patients in the transapical stratum. These are the 4 lower curves labeled "Neurologic event" in each of the individual competing risks graphs (Figure E4, $A-D$ ), but on an expanded vertical scale. Within each subgroup, the fractions alive without a neurologic event, dead before a neurologic event, and sustaining a neurologic event are shown individually in Figure E4, $A$ - $D$. Number of patients at risk are denoted below the horizontal axis at 6-month intervals.

Risk factors for late neurologic events were dominated by "non-TF-TAVR candidate" (more severe, generalized atherosclerotic burden), history of antecedent stroke or TIA, and more advanced functional disability, with similar adverse impacts across both the TAVR and AVR treatment arms (Figure 3, $C$ to $D$ ). (6) Preoperative rhythm (or surrogates for AF) could not be linked to neurologic events. (7) Using competing hazards (death or sustaining a neurologic event) analysis, the AVR subset in the TF stratum had the most favorable outcome at 2 years, that is, being alive and free from a neurologic event, whereas those in the TATAVR subgroup had the highest rate of adverse events (Figure 4). (8) Neurologic events after TAVR or AVR carried different mortality consequences (Figure 5).

The "non-TF candidate" risk factor represents a composite marker that coalesces patient and disease information. To explore this further, we repeated the bagging analysis with the preoperative "non-TF candidate" variable suppressed: the variables identified by bagging remained the same except for previous CABG (positive coefficient) entering the late hazard phase. Thus, the higher neurologic event rates in the TA stratum compared with the TF stratum were due to patient-related and/or disease-related factors, rather than some intrinsic feature of the TA-TAVR approach per se. There is no evidence available today, however, indicating that the risk of stroke during TF-TAVR will be lower using the next generation systems. Preliminary observations from the Prevail EU Registry in Europe using the Edwards SAPIEN XT valve and $18 \mathrm{Fr}$ and $19 \mathrm{Fr}$ TF NovaFlex delivery systems (Edwards Lifesciences) showed no drop in the incidence of stroke compared with the historical rates using the original SAPIEN valve.

Although TAVR is associated with higher early neurologic event rates, the responsible mechanism(s) remain unclear. Possible causes of brain injury include hypotension or hypertension, hemorrhagic events possibly related to anticoagulation, and emboli. ${ }^{16-18}$ The most likely cause of the early phase strokes and TIAs is gaseous or particulate cerebral embolization, probably owing to manipulation of guide wires and catheters across tightly stenotic aortic valves and the aortic arch or the preparatory balloon aortic valvuloplasty (BAV). ${ }^{18}$ Expansion of the TAVR bioprosthetic frame resulting in traumatic distortion of native valve elements and creating niduses of stagnant blood flow in the small remaining space within the aortic root may be a source of particulate debris or thromboemboli. ${ }^{16,18}$ Transcranial Doppler monitoring of middle cerebral artery flow to detect microembolic signals during TAVR showed showers during the preparatory BAV and valve delivery phases of TATAVR ${ }^{19}$ and during wire manipulation in the arch and valve implantation phases in either TA or TF cases, ${ }^{20}$ but there was no correlation between transcranial Doppler abnormalities and clinical neurologic status or National Institutes of Health Stroke Score. 


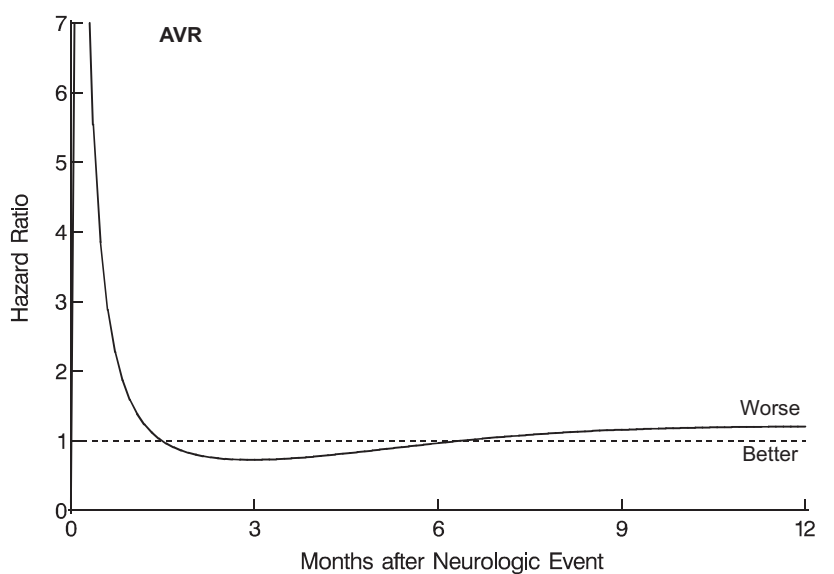

A

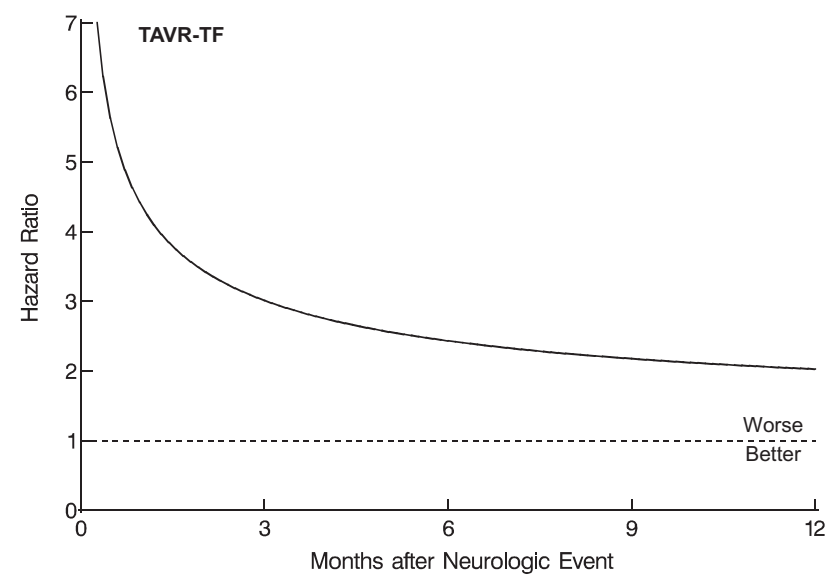

B

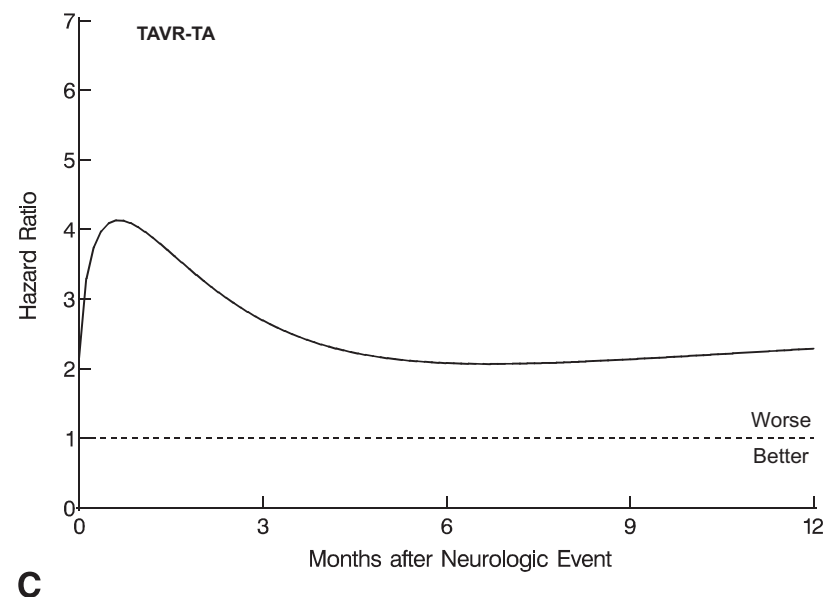

FIGURE 5. Hazard ratio of observed mortality after a neurologic event to expected mortality had the event not occurred. These hazard ratios represent the distillation of observed and expected hazard functions for death with and without a neurologic event depicted in Figure E5. A, Hazard ratio for surgical open aortic valve replacement $(A V R)$ treatment arm over the first postoperative year, based on Figure E5, $A$ and $B$. B, Hazard ratio for the transfemoral transcatheter aortic valve replacement (TF-TAVR) treatment arm over the first postoperative year, based on Figure E5, $C$ and $D$. C, Hazard ratio for the transapical transcatheter aortic valve replacement $(T A-T A V R)$ treatment arm over the first postoperative year, based on Figure E5, $E$ and $F$.

Avoiding the BAV step, ${ }^{21}$ not passing guide wires or catheters across the arch, ${ }^{22}$ and temporary percutaneous embolic deflectors ${ }^{23}$ are being investigated to determine whether intraprocedural cerebral embolism can be reduced. Concern has been spawned by reports showing that postoperative diffusion-weighted magnetic resonance imaging scans show acute ischemic lesions diffusely scattered throughout all territories of the brain in a majority of patients undergoing either TA or TF-TAVR (68\%-90\%) and to a lesser degree after AVR in lower surgical risk patients $(8 \%-48 \%)$ without high stroke rates, ${ }^{24-28}$ These abnormalities have been termed "clinically silent," but their potential impact on neurocognitive and higher memory function remain unclear. ${ }^{29-32}$ The total ischemic burden assessed by new diffusion-weighted magnetic resonance imaging ischemic lesions correlated with neurocognitive impairment, but the findings were mixed. ${ }^{30-32}$ Sophisticated neuropsychologic and cognitive testing in larger numbers of patients undergoing TAVR and AVR correlated with long-term neurobehavioral outcome is necessary. ${ }^{17}$

One-half of neurologic events occurred late after the procedure, indicating how stroke-prone this elderly patient population is. Measures aimed at reducing this risk may include intensified antiplatelet therapy before TAVR and/or more aggressive anticoagulation after TAVR (possibly including indefinite anticoagulation with warfarin or a direct factor Xa or thrombin inhibitor ${ }^{16-18}$ ), although this remains to be defined by prospective randomized clinical trials. ${ }^{17}$ Being assigned to the TA stratum was the strongest determinant of neurologic events late postoperatively, and the adverse impact of this risk factor cut equally across both TAVR and AVR treatment arms (Figure 3, $C$ and $D$ ). This late risk may not decline in the future unless more stringent patient selection excludes persons with extensive generalized arteriosclerosis from being considered TAVR candidates. 
This study was conducted using large-caliber TAVR delivery systems predominantly in institutions without previous TAVR experience in old and sick patients. Therefore, there is room for improvement, including technical refinements, more astute patient selection, improved procedural methods, and better anticoagulant and antiplatelet drug therapy. ${ }^{16-18}$ Concerns about stroke during TAVR remain, ${ }^{3,16-18,24-28}$ which will mandate more randomized trials as only prospective controlled comparisons will determine conclusively the risk/benefit ratio of TAVR compared with open AVR. ${ }^{16,17}$ Future investigations will also determine whether device improvements, for example, smaller TAVR delivery systems, embolic protection devices, better patient selection, and increased operator experience, can reduce the incidence of neurologic complications after TAVR. ${ }^{17,18}$ Until that information becomes available, the 2011 American College of Cardiology-Society of Thoracic Surgeons recommendations for "rational dispersion" of TAVR technology should be enforced, ${ }^{33}$ lest we repeat the health care dilemma created by misuse and overuse of carotid artery stenting. ${ }^{34}$

\section{Limitations}

These implications cannot be extrapolated to individuals other than the selected patients in the PARTNER cohort A trial, which represents the highest decile of STS risk for isolated AVR in the United States. ${ }^{2}$ Forty-seven $(7 \%)$ patients sustained 49 neurologic events, limiting the statistical inferences that can be drawn. Only 29 major strokes occurred, and the incidence was similar in the TAVR and AVR arms. The analyses in the TA patient strata (both TAVR and AVR) are limited by small sample and event sizes; therefore, assumptions and clinical relevance inferred from the TA patient analyses should be viewed as only preliminary. Limitations inherent in assessment of neurologic injury constitute weaknesses that will be rectified in the PARTNER-2 trial. Incomplete information concerning anticoagulation and antiplatelet therapy was another limitation. Neurologic events were possibly underestimated in AVR patients owing to more prolonged sedation. Finally, patients were followed up for only a minimum of 1 year, which mandates future long-term evaluation focused on valve durability and the late occurrence of valve-related adverse events, including neurologic complications.

\section{References}

1. Leon MB, Smith CR, Mack M, Miller DC, Moses JW, Svensson LG, et al. Transcatheter aortic-valve implantation for aortic stenosis in patients who cannot undergo surgery. N Engl J Med. 2010;363:1597-607.

2. Smith CR, Leon MB, Mack MJ, Miller DC, Moses JW, Svensson LG, et al. Comparison of transcatheter and surgical aortic valve replacement for aortic stenosis in patients at high-risk for operation. N Engl J Med. 2011;364:2187-98.

3. Brown JM, O'Brien SM, Wu C, Sikora JA, Griffith BP, Gammie JS. Isolated aortic valve replacement in North America comprising 108,687 patients in 10 years: changes in risks, valve types, and outcomes in The Society of Thoracic Surgeons National Database. J Thorac Cardiovasc Surg. 2009;137:82-90.

4. Shroyer ALW, Coombs LP, Peterson ED, Eiken MC, DeLong ER, Chen A, et al. The Society of Thoracic Surgeons: 30-day operative mortality and morbidity risk models. Ann Thorac Surg. 2003;75:1856-65.

5. Wolman RL, Nussmeier NA, Aggarwal A, Kanchuger MS, Roach GW, Newman MF, et al. Cerebral injury after cardiac surgery: identification of a group at extraordinary risk. Multicenter Study of Perioperative Ischemia Research Group (McSPI) and the Ischemia Research Education Foundation (IREF) Investigators. Stroke. 1999;30:514-22.

6. Sotaniemi KA. Cerebral outcome after extracorporeal circulation. Comparison between prospective and retrospective evaluations. Arch Neurol. 1983;40:75-7.

7. Tarakji KG, Sabik JF 3rd, Bhudia SK, Batizy LH, Blackstone EH. Temporal onset, risk factors, and outcomes associated with stroke after coronary artery bypass grafting. JAMA. 2011;305:381-90.

8. Bach DS, Siao D, Girard SE, Duvernoy C, McCallister BD Jr, Gualano SK. Evaluation of patients with severe symptomatic aortic stenosis who do not undergo aortic valve replacement: the potential role of subjectively overestimated operative risk. Circ Cardiovasc Qual Outcomes. 2009;2:533-9.

9. Gummert JF, Funkat AK, Beckmann A, Ernst M, Hekmat K, Beyersdorf F, et al. Cardiac surgery in Germany during 2010: a report on behalf of the German Society for Thoracic and Cardiovascular Surgery. Thorac Cardiovasc Surg. 2011; 59:259-67.

10. Thomas M. The global experience with percutaneous aortic valve replacement JACC Cardiovasc Interv. 2010;3:1103-9.

11. Rodés-Cabau J, Webb JG, Cheung A, Ye J, Dumont E, Feindel CM, et al. Transcatheter aortic valve implantation for the treatment of severe aortic stenosis in patients at very high or prohibitive surgical risk: acute and late outcomes of the multicenter Canadian experience. J Am Coll Cardiol. 2010;55:1080-90.

12. Redlich K, Khaladj N, Peterss S, Pichlmaier M, Shrestha M, Hoy L, et al. Conventional aortic valve replacement in patients with concomitant coronary artery disease and previous coronary artery bypass grafting in the era of interventional approaches. Eur J Cardiothorac Surg. 2011;40:455-62.

13. El Bardissi AW, Shekar P, Couper GS, Cohn LH. Minimally invasive aortic valve replacement in octogenarian, high-risk, transcatheter aortic valve implantation candidates. J Thorac Cardiovasc Surg. 2011;141:328-35.

14. Schaff HV. Transcatheter aortic-valve implantation-at what price? New Engl J Med. 2011;364:2256-8.

15. Johnston DR, Atik FA, Rajeswaran J, Blackstone EH, Nowicki ER, Sabik JF, et al. Outcomes of less invasive J-incision approach to aortic valve surgery. $J$ Thorac Cardiovasc Surg. 11 January 2012 [Epub ahead of print].

16. Amat-Santos IJ, Rodés-Cabau J, Urena M, DeLarochellière R, Doyle D, Bagur R, et al. Incidence, predictive factors, and prognostic value of new-onset atrial fibrillation following transcatheter aortic valve implantation. J Am Coll Cardiol. 2012; 59:178-88.

17. Daneault B, Kirtane AJ, Kodali SK, Williams MR, Genereux P, Reiss GR, et al Stroke associated with surgical and transcatheter treatment of aortic stenosis: a comprehensive review. J Am Coll Cardiol. 2011;58:2143-50.

18. Tay ELW, Gurvitch R, Wijesinghe N, Nielispach F, Wood D, Cheung A, et al. A high-risk period for cerebrovascular events exists after transcatheter aortic valve implantation. JACC Cardiovasc Interv. 2011;4:1290-7.

19. Drews T, Pasic M, Buz S, Unbehaun A, Dreysse S, Kukucka M, et al. Transcranial Doppler sound detection of cerebral microembolism during transapical aortic valve implantation. Thorac Cardiovasc Surg. 2011;59:237-42.

20. Szeto WY, Augoustides JG, Desai ND, Moeller P, McGarvey ML, Walsh E, et al. Cerebral embolic exposure during transfemoral and transapical transcatheter aortic valve replacement. J Card Surg. 2011;26:348-54.

21. Grube E, Naber C, Abizaid A, Sousa E, Mendiz O, Lemos P, et al. Feasibility of transcatheter aortic valve implantation without balloon pre-dilation. JACC Cardiovasc Interv. 2011;4:751-7.

22. Bagur R, Rodés-Cabau J, Doyle D, De Larochellière R, Villeneuve J, Bertrand OF, et al. Transcatheter aortic valve implantation with "No Touch" of the aortic arch for the treatment of severe aortic stenosis associated with complex aortic atherosclerosis. J Card Surg. 2010;25:501-3.

23. Nietlispach F, Wijesinghe N, Gurvitch R, Tay E, Carpenter JP, Burns C, et al. An embolic deflection device for aortic valve interventions. JACC Cardiovasc Interv. 2010;3:1133-8.

24. Kahlert P, Knipp SC, Schlamann M, Thielmann M, Al-Rashid F, Weber M, et al Silent and apparent cerebral ischemia after percutaneous transfemoral aortic valve implantation: a diffusion-weighted magnetic resonance imaging study. $\mathrm{Cir}$ culation. 2010;121:870-8. 
25. Adams HP Jr. Ischemic cerebrovascular complications of cardiac procedures. Circulation. 2010;121:846-7.

26. Astarci P, Glineur D, Kefer J, D'Hoore W, Renkin J, Vanoverschelde J-L, et al. Magnetic resonance imaging evaluation of cerebral embolization during percutaneous aortic valve implantation: comparison of transfemoral and trans-apical approaches using Edwards Sapien valve. Eur J Cardiothorac Surg. 2011;40:475-9.

27. Rodés-Cabau J, Dumont E, Boone RH, Larose E, Bagur R, Gurvitch R, et al. Cerebral embolism following transcatheter aortic valve implantation: comparison of transfemoral and transapical approaches. J Am Coll Cardiol. 2010;57:18-28.

28. Ghanem A, Müller A, Nähle CP, Kocurek J, Werner N, Hammerstingl C, et al. Risk and fate of cerebral embolism after transfemoral aortic valve implantation: a prospective pilot study with diffusion-weighted magnetic resonance imaging. J Am Coll Cardiol. 2010;55:1427-32.

29. Omran H, Schmidt H, Hackenbroch M, Illien S, Bernhardt P, von der Recke G, et al. Silent and apparent cerebral embolism after retrograde catheterisation of the aortic valve in valvular stenosis: a prospective, randomized study. Lancet. 2003; 361:1241-6.

30. Knipp SC, Matatko N, Schlamann M, Wilhelm H, Thielmann M, Forsting M, et al. Small ischemic brain lesions after cardiac valve replacement detected by diffusion-weighted magnetic resonance imaging: relation to neurocognitive function. Eur J Cardiothorac Surg. 2005;28:88-96.

31. Knipp SC, Matatko N, Wilhelm H, Schlamann M, Thielmann M, Lösch C, et al. Cognitive outcomes three years after coronary bypass surgery: relation to diffusion-weighted magnetic resonance imaging. Ann Thorac Surg. 2008;85: 872-9.

32. Barber PA, Hach S, Tippett LJ, Ross L, Merry AF, Milsom P. Cerebral ischemic lesions on diffusion-weighted imaging are associated with neurocognitive decline after cardiac surgery. Stroke. 2008;39:1427-33.

33. Homes DR Jr, Mack MJ. Transcatheter valve therapy: a professional society overview from the American College of Cardiology Foundation and The Society of Thoracic Surgeons. J Am Coll Cardiol. 2011;58:445-55.

34. Halm EA. Carotid stenting at the crossroads: practice makes perfect, but some may be practicing too much (and not enough). JAMA. 2011;306:1378-80.

\section{Discussion}

Dr John G. Byrne (Nashville, Tenn). Craig, this is a fantastic paper, superbly delivered. First, the results of the PARTNER trial show amazing results in both the traditional AVR as well as the TAVR groups. This well-designed and executed study documented the results using a first-generation large device in very elderly sick patients in which all adverse events were adjudicated by a CEC. We all congratulate you, Craig, and your coinvestigators on this historic trial. I have 3 questions.

Do you believe, as I do, that TAVR is here to stay? That open AVR, traditional AVR, for senile calcific AS is perhaps an endangered species? That in the future, open AVR will be reserved for cases of endocarditis, root disease, bicuspid disease and the very young? Given the amount of investment in this technology, both financial and intellectual, isn't it just a matter of time that TAVR will become the procedure of choice for most senile patients with calcific AS? Once SAPIEN and eventually CoreValve are approved by the Food and Drug Administration, how can surgeons remain involved? Will cardiologists own the TF approach and surgeons own the TA approach? How do we stay involved in the TF approach to this?

Dr Miller. Thank you, John, for those kind comments, which are very germane. The first question is related at preventing abuse and inappropriate use of TAVR. How do we prevent a runaway train, as what has happened in Germany where now 20\%-25\% or more of all AVRs are done percutaneously? This is a question that ultimately is going to be dictated by the payors, and we must also be mindful of the fact we don't even have medium- term valve durability data yet. TAVR is not going to be cost effective and affordable if these valves don't outlast most of the patients they are inserted in. Now, "a goodly amount of time" is rather short for a 95-year-old who is otherwise inoperable; it is completely different if you have a patient under 70 who has a very low surgical risk and should have a 15-20 year life expectancy. So we have to learn a lot more about the durability of percutaneous bioprosthetic valves before they are used in younger, healthier patients.

The proof of the pudding will actually be at the payor level; they are the only ones who can enforce how TEVAR is applied. Personally I am disappointed by what has happened in Germany. Here in the United States, we have no idea once one or both of these devices are approved by the FDA, but it is essential that a truly functional integrated heart valve team make sure that these complementary technologies-TAVR and surgical AVR-are used appropriately. The heart valve team and the payors must draw and enforce both lower and upper boundaries based on open surgical AVR risk and life expectancy to ensure reasonable use of TAVR. The lower boundary separates out the younger healthier patients who should undergo open AVR even if they demand a catheter percutaneous valve. The upper boundary is the line between utility and futility where we must learn to say "No." Many elderly, very sick patients with critical AS are dying of a host of other serious medical problems, which in the PARTNER Trial we call "Cohort C." They have extreme co-morbidities that overwhelm the potential benefits of TAVR; indeed, successful TAVR in these Cohort $\mathrm{C}$ patients may only prolong their suffering, and certainly is financially irresponsible for society.

I don't think open surgical AVR is an endangered species. There are many patients with severe AS out there we as surgeons have never seen who potentially can benefit from either TAVR or AVR, as the PARTNER Trial Cohort A results illustrate. Remarkably, in the PARTNER Cohort B (inoperable) New England Journal of Medicine paper in October 2010, the TAVR patients gained 1.9 quality years of life and it only cost \$US55,000 per QUALY, substantially less than the cost of dialysis per QUALY. So in the inoperable cohort TAVR is cost effective and provides meaningful rehabilitation. In the younger high risk operable patients, we have to work together in the heart valve team to determine whether TAVR or AVR offers the best option for each individual patient. I repeat that these are complementary treatment methods, not competitive. On the other hand, the transapical TA-TAVR approach may possibly be an endangered species since direct aortic access TAVR is now being explored in Europe; an upper mini-sternotomy or even high anterior right thoracotomy is better tolerated than a low left small thoracotomy, and there is no LV injury from the TA purse-string sutures. In the "TF first" PARTNER Cohort A trial, however, being judged a non-TF-TAVR candidate confered a higher risk patient to begin with, and an elevated risk of late neurologic complications.

We need strict patient selection. The way we and the other PARTNER sites have found this to work well is to make the entire process truly a team effort: In the preoperative decision-making phase, during the procedure (a surgeon and interventional cardiologist alternate being the primary operator in TF-TAVR cases at Stanford), and taking care of the patients after TAVR. Marty Leon will tell you himself that it is easier, more fun, and quicker 
to do a TA-TAVR case than a TF-TAVR long distance from the groin. So if you share the experience amongst your team and learn from each other, it actually can be synergistic. This takes mutual respect and mutual trust. It is very refreshing at this stage of my career to see cardiovascular surgeons and interventional cardiologists set aside our parochial self-interests and egos in order to work together for the patient's benefit.

Dr Byrne. Two more questions: Your study showed that the risk of stroke continues well after the procedure. What is the mechanism of continued stroke? Is it thrombus formation on the prosthesis or does calcium continue to break loose? We surgeons meticulously remove calcium, and in TAVR the calcium is left behind. Is it thrombus or calcium or both or just heavy atherosclerotic burden?

Finally, given that the risk of stroke continues most markedly between 1 month and 1 year after surgery, what approaches do you and your coinvestigators recommend to mitigate stroke? What is the role of dual antiplatelet therapy and cerebral protection devices?

Dr Miller. John, those are also very good questions. Given we used a large, second generation TAVR device and there was a steep learning curve in most of the PARTNER centers (only 6 of the 26 sites had any prior TAVR experience), there is ample room for improvement in patient selection, procedural expertise, and postoperative patient management.

Even though we can't prove it, the early neurologic events within the first few days were probably due to particulate cerebral embolization. There are several cerebral embolic protection deflectors and filters being developed that cover the arch vessels and may reduce this intraprocedural neurologic event risk. For the neurologic complications occurring later on, we have to remember that there is a high background neurologic event incidence in these very old, sick patients that none of us can change. It is unknown whether dual antiplatelet therapy or perhaps even warfarin or other anticoagulant drug administered over the long-term might reduce the late neurologic event rate. In the TAVR patients, there are many nooks and crannies remaining around the native calcified cusps which possibly may lead to stagnant flow with eventual thrombus formation around the SAPIEN valve. I don't believe embolization of calcific debris from the aortic cusps is a major factor late postoperatively. More likely micro-thrombus or platelet aggregates are forming in some of these blind niches around the percutaneous valve, which later can embolize to the brain. This is an open question, and an important one; there is hope for improvement in late risk of neurologic events as the antiplatelet and antithrombotic treatment regimens are refined.

Thank you.

Dr Irving Kron (Charlottesville, $\mathrm{Va}$ ). Craig, that was a great discussion, and I know that your heart and soul has been in this study. What amazed me was, frankly, the terrific results both for open AVR and the percutaneous device in the patients who had the TF approach. Do you think our risk adjustment scores are accurate?

Dr Miller. That is a great question, Irv. We must recall that the STS scores have never been completely validated out at this extreme end of the spectrum. The PARTNER cohort A patients constitute the highest STS risk decile, or the upper 10th percentile of STS risk, and there just haven't been enough of them in the STS database to be certain. To answer your question, based on our results from these 26 institutions, the STS expected operative risk score would appear to overestimate the observed open surgical AVR risk, but we do not have any certainty this would be true US-wide, from which the STS database comes. What would be the result of such a trial in a real world situation? We do not know, but were pleasantly surprised by the low death rates and neurologic event rates in both the AVR and the TAVR groups. This is especially true since data monitoring was prospective, monitored, audited, and an external Adverse Events Committee was looking over our shoulder. We couldn't hide anything in this study even if we had tried.

Dr Thomas W. Rice (Cleveland, Ohio). I found the mortality cost data interesting. If you receive an open AVR, the observed risk of death approaches the expected. If you receive a TAVR, the observed risk of death does not approach the expected. Do you have any insight?

Dr Miller. I do not know, Tom, but I speculate that perhaps the neurologic events in the AVR group may have been more severe cerebral insults such that more patients died right away. Or perhaps it reflects a difference in the philosophy and aggressiveness of the treating local physicians. Most late neurologic events happened far away from the PARTNER TAVR centers; if a patient after TAVR sustained a serious stroke the primary care physicians and the family may have been more nihilistic, saying something like, "well, we gave it the best shot, but let's not let Granny suffer anymore." We just don't know, but it is interesting.

Dr Hartzell V. Schaff (Rochester, Minn). Craig, I want to follow up on a question that John Byrne asked you, which had to do with antiplatelet therapy. I believe that the transcatheter patients were all treated with dual antiplatelet therapy for 6 months or a year. Do you think that the lack of that in a surgical group had anything to do with the difference in the late risk of stroke?

Dr Miller. That is an excellent question, Hartzell. Dual antiplatelet therapy was recommended postoperatively for all the TAVR patients, but it was only a recommendation. How strictly it was adhered to and for how long is poorly characterized. One large enrolling center had very few early neurologic complications in the TAVR patients; they used aggressive antiplatelet and anticoagulant therapy. Antiplatelet therapy for the AVR patients was left to the discretion of the local surgeons, but I suspect most of them also received at least aspirin postoperatively. We need to unravel this dilemma more completely in the future, but most PARTNER investigators today now pay more assiduous attention to the antiplatelet and anticoagulation regimen, even including starting antiplatelet drugs immediately before the TF procedures. Since there was no difference in neurologic complication risk between the TAVR and AVR subgroups during the late hazard phase, I do not think antiplatelet therapy had any influence on neurologic event risk. Recall that late postoperatively the strongest determinant of neurologic events was being judged to be a "non-TF candidate," which cut equally across the TAVR subgroup as well as the AVR subgroup. This is a patient-related and disease-related risk factor, which cannot be changed. 


\section{APPENDIX E1 \\ Detailed Methods}

Study design, inclusion and exclusion criteria, study organization, study sites, principal investigators, follow-up, and the SAPIEN transcatheter heart valve and delivery systems have all been previously reported. ${ }^{\mathrm{E} 1 \mathrm{E} 2}$

Patient sample. The PARTNER trial enrolled only patients with severe AS and advanced cardiac symptoms (New York Heart Association class $\geq$ II). Patients were at high-risk for conventional AVR based on coexisting conditions predicting a risk of death by 30 days after operation of $15 \%$ or more. An STS mortality risk score ${ }^{\mathrm{E} 3}$ of $10 \%$ or more was used as a floor for risk stratification, but the final determination of operative risk was made by the local surgeons after dialogue on the biweekly PARTNER patient selection conference call.

As previously reported, ${ }^{\mathrm{E} 1, \mathrm{E} 2}$ between May 11, 2007, and August 28, 2009, from among the 3105 patients screened, $34 \%$ were ultimately randomized in PARTNER, with 699 $(23 \%)$ being in the high operative risk cohort A limb. Twenty-six sites participated, including 22 in the United States and 3 in Canada; only 6 sites had previous TAVR experience. Because this study focuses on neurologic procedural complications, only the 657 patients who underwent assigned treatment ("as treated," or AT) were considered further (42 patients excluded ${ }^{\mathrm{E} 2}$ ). An additional 2 patients were excluded from analysis because they never received their assigned treatment: one refused open AVR and sustained a stroke during palliative BAV; the second had a stroke before AVR, deteriorated clinically, and died. Roll-in cases were also excluded, among which 4 patients had neurologic events. Nine (2.6\%) attempted TAVR cases had to be aborted or converted, and multiple valves ("valvein-valve") were acutely necessary for various exigencies in 7 TAVR patients.

Two cross-over patients experienced neurologic events: one patient (TF stratum) who was randomized to TAVR was converted from TF-TAVR to TA-TAVR on the table when safe peripheral access could not be achieved and sustained a major stroke on day 1 . The other patient (TA stratum, randomized to open AVR) underwent redo sternotomy and attempted AVR but the procedure was aborted when a totally calcified ascending aorta and arch were encountered. Six weeks later, this patient was allowed under Canadian Compassionate Use provisions to cross over and undergo TA-TAVR; he had a major stroke 11 months later. These 2 patients were considered according to initial treatment assigned in this analysis. A sensitivity analysis was performed by rerunning the hazard function calculations after moving the second individual from the AVR group to the TA-TAVR group: The coefficients in the model did not change substantially, indicating this aberration did not affect the conclusions.
Study design, oversight, and data management. The PARTNER trial study design and data management practices have been described previously. ${ }^{\text {E1,E2 }}$ Patients were randomized according to a computer-generated scheme, blocked separately at each site and for each randomized trial cohort. In the high-risk but operable cohort A, patients were first categorized anatomically to either the TF-TAVR or TA-TAVR stratum and then were randomized within their assigned category to undergo either TAVR or AVR. For data analysis of the "AT" patients, time 0 started with induction of anesthesia. The trial was approved by institutional review boards at each site. Written informed consents were obtained for all patients.

The CONSORT patient flow diagram for the AT patients analyzed is shown in Figure E1. Six hundred fifty-seven patients among the 699 individuals randomized in ITT received designated treatment ("as treated"), 461 in the TF and 196 in the TA stratum. Follow-up closing date was September 21, 2010, which corresponded to when every ITT patient had been followed up for a minimum of 1 year and 1 month. Four hundred eighty-four patients remained at risk at 1 year and 124 patients at 2 years (111 were in the TF stratum). It is important to note that only the overall pooled TAVR versus AVR and the TF stratum (TF-TAVR versus AVR) comparisons were adequately powered to detect statistical noninferiority. No comparisons were adequately powered to detect superiority. The data reflect the clinical database as of May 6, 2011.

This study was designed by the sponsor and executive committee, which included the 2 co-principal investigators, 3 interventional cardiologists, and 3 cardiovascular surgeons. The sponsor funded the trial and participated in selection and management of the sites, collection of the data, and data monitoring. The executive committee met in person every 6 to 8 weeks to monitor all aspects of trial conduct. The PARTNER Stroke Writing Group had unrestricted access to the data after the database was locked, performed all data analyses, prepared all drafts of the manuscript, and attests to the integrity of the trial and the completeness and accuracy of the reported observations.

Neurologic end points. The Food and Drug Administration-approved PARTNER investigational device exemption protocol for assessment of neurologic events was limited in comprehensiveness.

Central nervous system (neurologic) events. A TIA was defined as a focal neurologic event that was fully reversible in less than 24 hours in the absence of any new imaging findings of infarction or other primary medical cause (eg, hypoglycemia or hypoxia). A stroke was defined as a focal neurologic deficit lasting 24 hours or more or a focal neurologic deficit lasting less than 24 hours with imaging findings of acute cerebral infarction or hemorrhage. Stroke was further classified as ischemic, hemorrhagic 
(epidural, subdural, subarachnoid), or ischemic with hemorrhagic conversion. It was also determined if the event was procedure or bioprosthetic device related after review of available source documents and brain imaging reports (2 neurologic events were judged to be device-related in TFTAVR patients). Major and minor stroke were subsequently distinguished through a CEC-adjudicated retrospective, unblinded analysis of neurologic events. A minor stroke was defined as an event associated with a modified Rankin Scale of 0 or 1 at 30 days or longer after the event or a National Institutes of Health Stroke Scale score of 0 . This was determined by review of the source documentation surrounding the stroke, including but not limited to progress notes, consult notes, discharge summaries, and follow-up clinic notes. If National Institutes of Health Stroke Scale information was available, this was incorporated into the adjudication. A major stroke was defined as a modified Rankin Scale score of 2 or more at 30 days or longer after the event.

The CEC could not judge the severity of the stroke in 6 cases and did not comment on severity of stroke when adequate source materials in their opinion were not available; these cases were examined by the PARTNER Trial Executive Committee and a collective decision made regarding severity of stroke. If the severity of neurologic injury was unclear, the default decision was to label the event a major stroke to be conservative. Two investigators (D.C.M. and M.J.M.) reviewed the CEC summaries and all available source documents for the 47 patients who sustained a neurologic event to corroborate the CEC and executive committee's decisions. No irreconcilable discordance with the CEC adjudications was encountered.

\section{Data Analysis and Detailed Statistical Methods}

Categorical variables were compared using Fisher's exact test. Continuous variables are presented as means $( \pm 1$ standard deviation) and compared using the Student $t$ test. When the data were markedly skewed, continuous variables are also presented as median with interquartile range. Data analysis was based on the "as treated" or AT principle. Time-to-event analyses were based on all available follow-up data that extended beyond 2 years. All analyses were performed using SAS statistical software (SAS version 9.2; SAS Institutes, Inc, Cary, NC). Both nonparametric (actuarial) and parametric estimates of neurologic event occurrence are presented with asymmetric $68 \%$ confidence intervals, comparable to \pm 1 standard error. We also present nomograms of the multivariable analyses (risk-adjusted solutions of the multivariable equation) for illustration. To enhance the certainty of the estimates, the "Berkson rule" of drawing the actuarial curves out until about $10 \%$ of cases remained alive without the event was employed; thereafter, what is known as the "completion effect" kicks in (the larger the original sample size the longer it takes for the completion effect to take hold, so for very large studies one can extend the actuarial curves much longer out). In general, the parametric method used in this manuscript is less sensitive to completion effects than are nonparametric actuarial (eg, Kaplan-Meier) methods. The parametric models were carried out to 2 years except for the TA stratum, where limited follow-up only made 1 -year estimates meaningful (Figure E1).

End points. The main focus of this analysis was on timerelated central neurologic events, 49 of which occurred in 47 patients. Although we explored repeated events, the analyses were time to the first neurologic event. As a secondary analysis, we estimated risk of major stroke, but the smaller numbers $(n=29)$ precluded meaningful statistical analyses; furthermore, our ability to determine accurately the severity of the neurologic damage was limited in the PARTNER-I trial. No reliable information was available regarding residual permanent disability after a stroke. We then placed these neurologic events into the context of the ongoing competing risk of death before a neurologic event occurs. Finally, we examined death after a neurologic event.

Missing values. A number of variables examined in multivariable analyses had missing values. We used 5-fold multiple imputation ${ }^{\mathrm{E} 4}$ using a Markov Chain Monte Carlo technique to impute missing values (SAS PROC MI). In multivariable modeling, for each imputed complete data set, we have estimated the regression coefficients and their variance-covariance matrix. Then following Rubin, ${ }^{\mathrm{E} 4}$ we combined estimates from the 5 models (SAS PROC MIANALYZE) to yield final regression coefficient estimates, the variance-covariance matrix, and $P$ values.

Time-varying instantaneous risk (hazard function) and occurrence of neurologic events. Nonparametric analyses of neurologic events overall and in each of the 2 treatment arms revealed an early high-risk periprocedural phase followed by a lower persistent occurrence of events for as long as the patients had been monitored. Two analyses were performed to determine occurrence of neurologic events as a function of time, define hazard functions, and identify incremental risk factors. The first was freedom from a neurologic event (47 events in 47 patients, ordinary time-to-event analysis), which was assessed nonparametrically using the Kaplan-Meier estimator and parametrically using a multiphase hazard model. ${ }^{\mathrm{E} 5}$ The parametric model was used to resolve a number of phases of instantaneous risk of the event (hazard function) and to estimate shaping parameters. In most cases, 2 hazard phases were resolved; the sum of these is the overall hazard. The second approach included all neurologic events, 49 in 47 patients, treated as repeated outcomes. Nelson's ${ }^{\text {E6,E7 }}$ cumulative event function was used to obtain nonparametric estimates. The cumulative number of events per patient was also estimated by multiphase hazard function methodology to 
obtain instantaneous risk of the event (hazard function). ${ }^{\text {E5 }}$ This manuscript focuses on the first approach: the initial neurologic event for each patient.

Preprocedural factors associated with early and late neurologic events. Because 2 distinct phases of risk were identified, it was likely that there were different risk factors or mechanisms responsible for each phase. One caveat is that with only 47 patients experiencing events (effective sample size), the ability to identify these factors was limited. We therefore performed a sequential analysis. The first only considered preprocedural factors common to both treatments, TAVR and AVR. No interaction terms were considered at this stage (factors that may be more strongly related to risk in 1 group than the other). The method to search for possible interacting factors that modulate risk in 1 treatment arm more or less than in the other arm is described below. In the multivariable analysis, factors modulating both hazard phases were considered simultaneously. Early risk factors were those found to increase the area beneath the early peaking hazard phase, and late risk factors increased the level of underlying late constant hazard. Within each hazard phase, we assumed proportional hazards, but because the 2 hazard phases are operative across all time, this produces overall a nonproportional hazard model. Such a model is particularly appropriate for strongly time-varying hazard, as is evident for neurologic events in this study.

Preoperative variables considered in the analysis are listed in Table E6. Variable selection, with a $P$ value criterion for retention of variables in the model of .07 because of the small number of events, used a computerintensive machine learning "bagging" method (bootstrap aggregation). ${ }^{\text {E8,E9 }}$ This was a 4-step process. First, a patient was randomly selected from the original data set to begin a new data set. The original data set continued to be sampled until the new data set was $100 \%$ the size of the original. In general, about two-thirds of patients are unique using such a sampling-with-replacement method, and about one third are duplicate patients. Second, risk factors were identified using automated forward stepwise selection. Third, results of the variable selection were stored. These 3 steps were repeated 500 times. Finally, the frequency of occurrence of variables found in these analyses was ascertained and indicated the reliability of each variable (aggregation step). It is generally thought that variables with bootstrap reliability of $50 \%$ or greater should be retained in the model. As will be noted, 1 factor with a $P$ value $<.1$ was retained in addition. No other factor was found to be close in reliability. An interpretation of reliability from such an analysis is that it represents the probability that the $P$ value is less than .07 .

Intraprocedure-specific factors associated with early and late neurologic events. In addition to the general preoperative factors, intraprocedural risk factors specific to each of the 2 procedures might influence stroke risk (Table E7). Each procedure group was analyzed separately to allow clues to emerge linking possible preoperative factors that are specific to each treatment arm. Generally, separate analyses such as these make it easier to detect possible interaction terms than forming the product of procedure with each variable in the analysis. In the TAVR arm, the only intraprocedural variable that was statistically significant was TA stratum, which increased risk during the late phase. This analysis strongly suggested that smaller native aortic valve area or higher mean transvalvular gradient might possibly be additional preprocedural risk factors. In the AVR arm, no procedural variable was statistically significant. Aortic valve size was not identified as a risk factor for neurologic events after AVR.

Variables specific to the procedure itself, as well as preprocedural variables, were analyzed using the same machine learning methodology previously described for preprocedural factors. Some variables could not be analyzed reliably, including valve embolism (TAVR group) because of too few events associated with this uncommon occurrence.

Pre-procedural risk factors for neurologic events specific to the treatment procedures. Analysis of predictors of neurologic events in each procedure arm suggested there may be preprocedural risk factors, particularly related to native aortic valve size, that may be specific for each of the treatment arms - so-called interaction terms in analysis of the pooled procedures. On the basis of the separate analyses of each treatment arm, we formed interaction terms with treatment to determine the following: (1) if there are risk factors for 1 and not the other procedure and (2) if strength of risk factors differed significantly between the arms. We confined our search for interaction terms to those related to the 2 procedures, not interaction among other risk factors, even though there is controversy among statisticians about how to express interaction terms such as this for continuous variables. We show that native valve size is only relevant to the TAVR arm, meaning that the factor is not relevant to the AVR arm (coefficient is 0). This is similar to how other variables that were not statistically significant were managed.

Further refinement of the model of risk factors for neurologic event and taking into account patient size. Examining variables reflecting patient size might potentially be particularly relevant to understanding the native aortic valve area risk factor in the TAVR group. In addition, the ratio of annulus dimension to prosthesis dimension (recognizing that this measurement differs among heart valve devices) was examined. Indexed aortic valve area (AVAI, valve area divided by body surface area $\left[\mathrm{cm}^{2} / \mathrm{m}^{2}\right]$ ) and indexed and normalized bioprosthesis size were forced into the model along with interactions with procedure (AVR vs TAVR). AVAI within the TAVR group was not only 
statistically more significant in relationship to early neurologic events than unindexed AVA, the unindexed AVA variable then fell out of the model $(P>.3$, Table 2). Bioprosthesis size (absolute, indexed, and normalized to annulus) was not found to be associated with increased risk of neurologic events in either hazard phase, with or without interactions. Note that in Table 2 the variable TAVR in the early hazard phase when interactions are considered becomes an offset to the intercept term in the model.

Analysis of major stroke. We analyzed all neurologic events out of necessity owing to the relatively small number of events, but for completeness sake we attempted to depict the incidence and hazard of major stroke despite major constraints that limited our ability to discriminate precisely the severity of neurologic damage sustained. Of the 31 neurologic events after TAVR, 18 were adjudicated to be major strokes, as were 11 of the 16 events after AVR. Of note, a number of neurologic events could not be classified and arbitrary conservative default judgments were made (vide supra). We used the same multiphase hazard function methodology to estimate the hazard function for major strokes as used for all neurologic events (see Figure E3, $A$ to $C$ ).

Competing risks of death and neurologic event. Actuarial and parametric estimates of the rate of neurologic event rates describe what happened for the entire patient sample being investigated; as time passes patients die (especially in this very old and sick patient cohort) and the question emerges: "What is the likelihood of experiencing a neurologic event given this strong competing risk of death?" Put more simply, what individual patients want to know is: "What are the odds if I am still alive I will be free from a neurologic event?" We explored this important and commonly asked question using competing hazard analysis where the following 2 mutually exclusive outcomes were considered as competing events: death before experiencing a neurologic event and sustaining a neurologic event.

A common interval was defined for analysis as the earliest of either death or neurologic event. Patients then transition from being alive without a neurologic event into 2 mutually exclusive states: neurologic event or death before such an event. Freedom from each event was estimated by the nonparametric product limit method. ${ }^{\text {E10 }}$ Variances of the estimates were based on Greenwood formula. ${ }^{\mathrm{E} 10} \mathrm{Be}$ cause of the difference in neurologic event risk according to TF or TA stratum, the TAVR group was analyzed according to "as stratified" approach, recognizing that the approach is indicative of more generalized atherosclerotic burden and not the TF or TA TAVR procedure per se. The results are illustrated for all 4 subsets of patients individually in Figure E4, $A$ to $D$, and summarized in Figure 4 in the main manuscript. An assumption in competing risks analysis is that censoring at times of transition into each state is "noninformative" (eg, timing of a neurologic event is not informative of time of death). This is an artificial assumption, and methods for managing "informative censoring" exist, but this is an area of statistical research, not of statistical practice.

"Mortality cost" of a neurologic event. Neurologic events carry not only a morbidity cost to the patient, but a mortality cost. Survival after a neurologic event was estimated nonparametrically by the Kaplan-Meier estimator and parametrically using a multiphase hazard model. ${ }^{\mathrm{E}}$ Time 0 was the time of the first neurologic event in the 47 patients. Mere depiction of survival after a neurologic event does not, however, answer the question of the mortality cost of such an event. For this, we need to estimate survival had the event not occurred. It is not correct to ask what survival was in patients who never experienced a neurologic event, because this assumes that ahead of time one knows who will experience such an event and who will not. Thus, we first estimated mortality before a neurologic event for all trial patients from the time of the procedure, stratified by AVR and within TAVR by TF and TA approaches. This is identical to the competing risks approach described above, where the death curve is "death before neurologic event," which we parametrically estimated using the multiphase hazard model. The next step was to use the parametric equation for survival before a neurologic event to generate a survival curve for each patient experiencing a neurologic event after the time the event occurred. This is known as conditional survival, which starts at $100 \%$ at the time of a neurologic event. We then computed the average of those 47 curves, which is the expected survival beyond the time of neurologic event had it never occurred. The third step is to compare the actual survival curve from time of neurologic event to the expected survival curve had the event not occurred (Figure E5, $A$ to $F$ ). Although the comparative results can be portrayed several different ways, difference in survival across time, hazard ratio across time, or decrement in lifetime (the area between the curves), in this manuscript, we present the hazard ratio across time (Figure 5, $A$ to $C$ ). A hazard ratio of 1.0 means observed and expected risk at that instant of time is identical.

Factors distinguishing TA-TAVR candidates from TFTAVR candidates. Non-TF-TAVR candidates had a higher risk of neurologic events than did TF candidates in the late constant hazard phase. We investigated preprocedural patient-related and disease-related factors that distinguished TA from TF stratum candidates. Multivariable logistic regression was used that employed the patient factors from Table E6. Bagging was used for variable selection. Table E2 verifies the clinical impression that arteriosclerotic vascular disease (equally peripheral and cerebrovascular disease) strongly characterized TA candidates. In addition, women were more likely to fit this profile as were those who had previous CABG. 
Interestingly, the lower the mean gradient the higher the likelihood of being a TA candidate. As anticipated, more recent time frame included more TA candidates owing to trial design (the TA limb enrollment started 1 year after the TF arm) and is reflected in shorter follow-up of these patients. A graphic depiction of this analysis is shown in Figure E2.

\section{E-References}

E1. Leon MB, Smith CR, Mack M, Miller DC, Moses JW, Svensson LG, et al. Transcatheter aortic-valve implantation for aortic stenosis in patients who cannot undergo surgery. $N$ Engl J Med. 2010;363:1597-607.

E2. Smith CR, Leon MB, Mack MJ, Miller DC, Moses JW, Svensson LG, et al. Comparison of transcatheter and surgical aortic valve replacement for aortic stenosis in patients at high-risk for operation. N Engl J Med. 2011;364:2187-98.
E3. O'Brien SM, Shahian DM, Filardo G, Ferraris VA, Haan CK, Rich JB, et al. The Society of Thoracic Surgeons 2008 cardiac surgery risk models: Part 2Isolated valve surgery. Ann Thorac Surg. 2009;88:23-42.

E4. Rubin DB. Multiple imputation for non-response in surveys. New York: John Wiley; 1997.

E5. Blackstone EH, Naftel DC, Turner ME Jr. The decomposition of time-varying hazard into phases, each incorporating a separate stream of concomitant information. J Am Stat Assoc. 1986;81:615-24.

E6. Nelson W. Theory and application of hazard plotting for censored failure data. Technometrics. 1972;14:945-66.

E7. Nelson W. Graphical analysis of system repair data. J Qual Technol. 1988;20: 24-35.

E8. Efron B, Tibshirani RJ. An introduction to the bootstrap. New York: Chapman and Hall/CRC; 1998.

E9. Breiman L. Bagging predictors. Machine learning. 1996;24:123-40.

E10. Andersen PK, Borgan O, Gill RD, Keiding N. Statistical models based on counting processes. Chapter 4. Nonparametric estimation. New York: Springer-Verlag; 1993. p. 176-331.

\begin{tabular}{c}
\hline Randomized $=699$ \\
TF $=492$ \\
TA $=207$ \\
\hline Not Treated \\
TF $=29$ \\
TA $=13$ \\
TAVR TF Converted to TA $=2$ \\
\hline
\end{tabular}
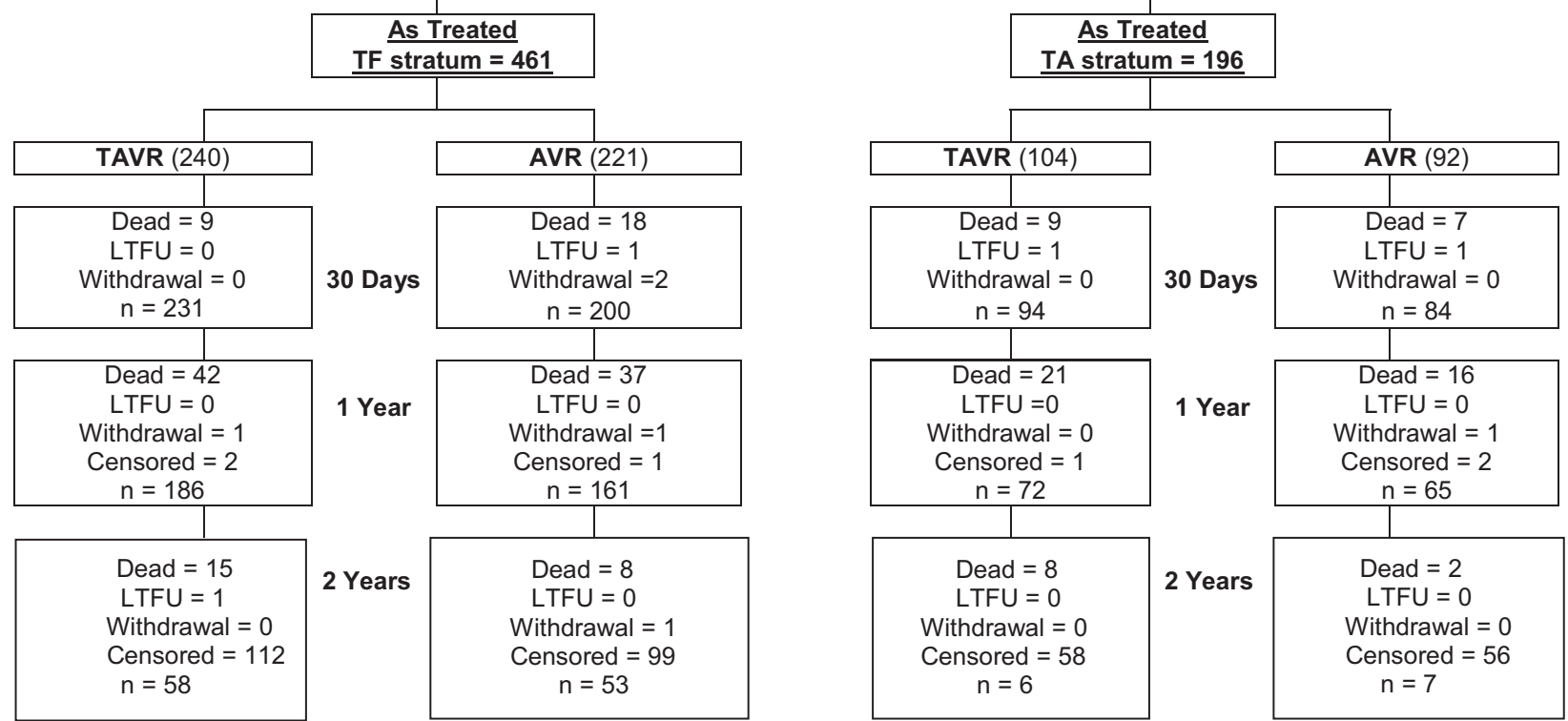

FIGURE E1. Patient flow CONSORT diagram: PARTNER cohort A ("as treated"). The analysis close date was at the end of 1 year's follow-up for all intent to treat patients (September 2010). At that time some of the patients had not reached 1 year according to the "as treated" timeline and are shown as censored in the 1 year counts. TF, Transfemoral; TA, transatrial; TAVR, transcatheter aortic valve replacement; $A V R$, aortic valve replacement; $L T F U$, long-term follow-up. 


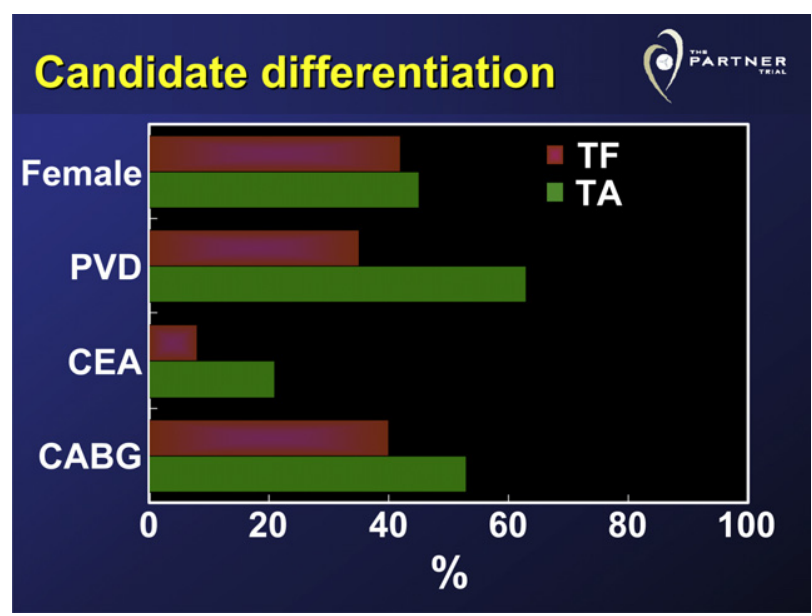

FIGURE E2. Patient characteristics segregating the transapical (TA) stratum from the transfemoral $(T F)$ stratum, which was carried out before randomization into the transcatheter aortic valve replacement (TAVR) or surgical aortic valve replacement $(A V R)$ treatment limbs. $P V D$, Peripheral vascular disease; $C E A$, carotid endarterectomy or stenting; $C A B G$, coronary artery bypass grafting. 


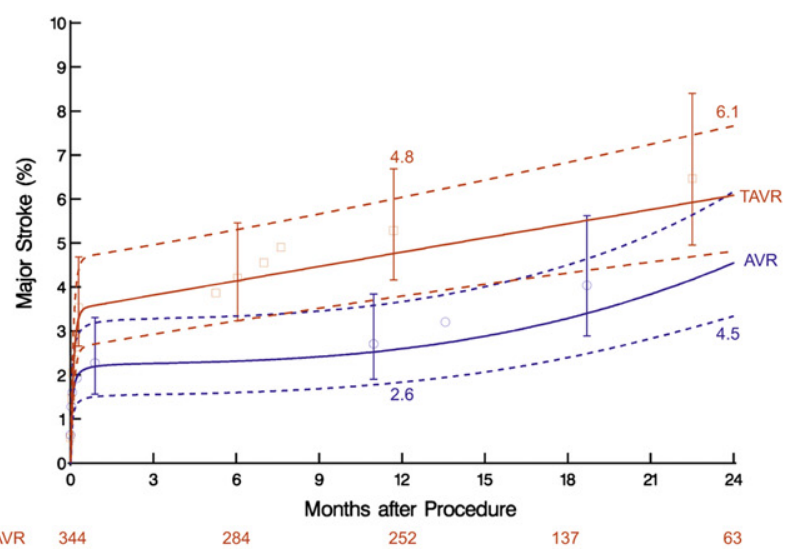

$\begin{array}{ll}\text { TAVR } & 344 \\ \text { AVR } & 313\end{array}$

A

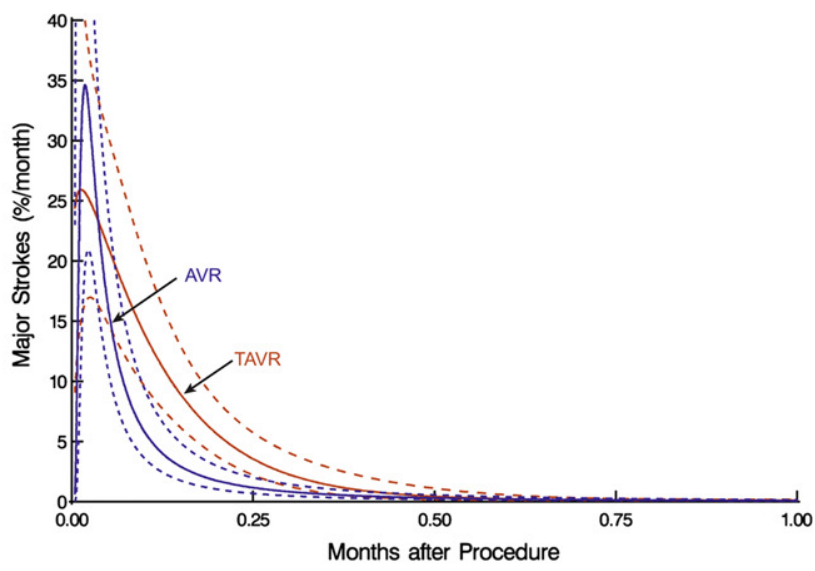

B

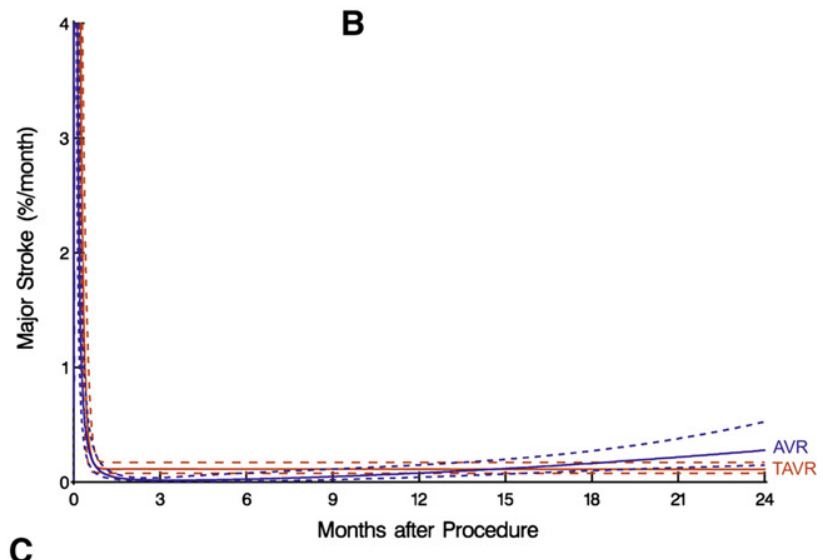

FIGURE E3. Major stroke after transcatheter aortic valve replacement (TAVR) and surgical aortic valve replacement (AVR). A, Occurrence of major stroke. Each symbol represents an event; vertical bars are confidence limits equivalent to \pm 1 standard error, and solid lines enclosed within dashed confidence bands indicating \pm 1 standard error represent parametric estimates. Numbers of patients at risk are denoted below the horizontal axis at 6-month intervals. The red curve and open squares depict the TAVR pooled (TA and TF strata) arm and the blue curve and open circles are for the surgical AVR pooled arm. Numbers within the plot are point estimates at 12 and 24 months. B, Instantaneous hazard of major stroke. Solid lines represent parametric estimates and are enclosed within asymmetric confidence bands equivalent to \pm 1 standard error. The red curve is for TAVR; the blue curve is for AVR. C, Instantaneous hazard of major stroke after TAVR or AVR. The depiction is identical to that in Figure E3, B, except that the vertical axis is expanded and the horizontal axis has been stretched to 24 months to depict later hazard. 

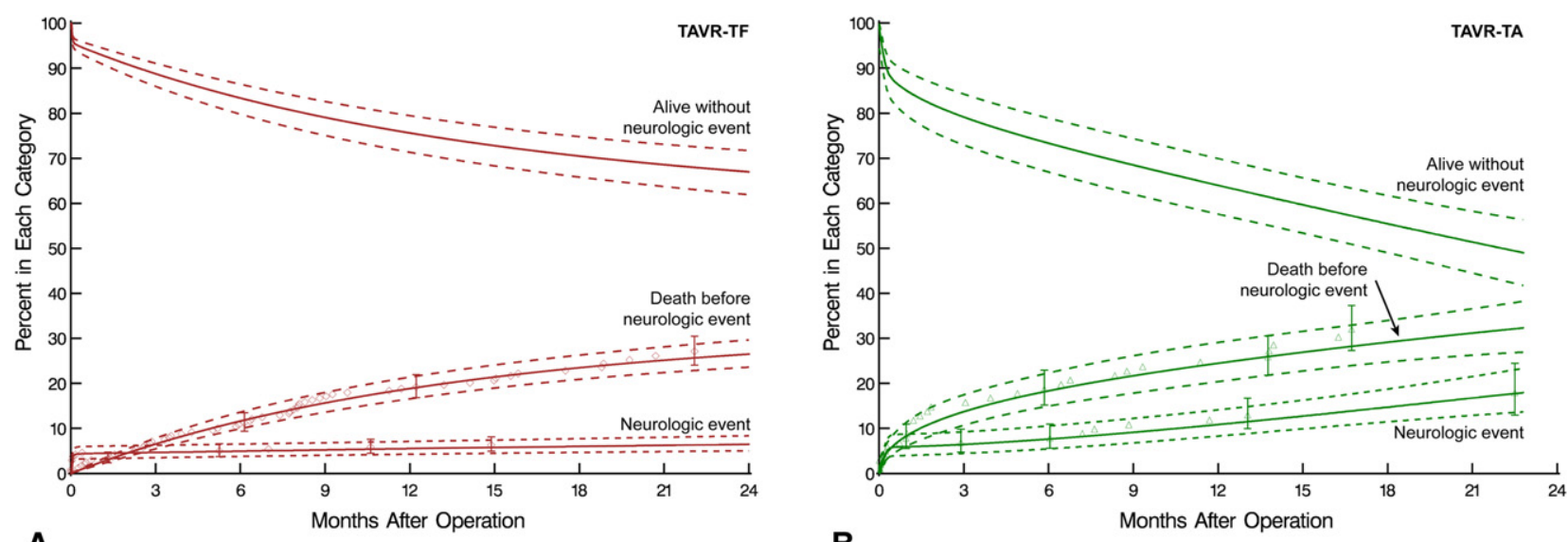

A

B
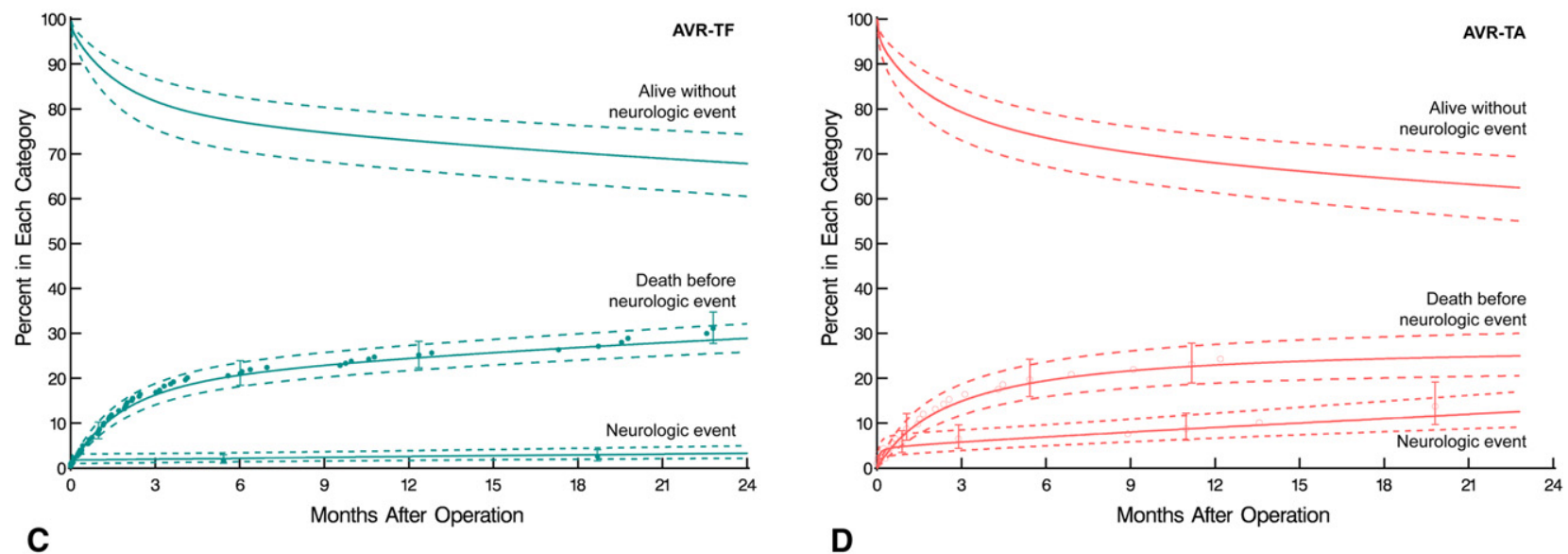

FIGURE E4. Competing risks of death or neurologic event after transcatheter aortic valve replacement (TAVR) and surgical aortic valve replacement $(A V R)$. At each time point, "alive without neurologic event" (or "event-free survival"), "death before neurologic event", and "neurologic event" add to $100 \%$. Each symbol represents an event; vertical bars are confidence limits equivalent to \pm 1 standard error, and solid lines enclosed within dashed confidence bands indicating \pm 1 standard error represent parametric estimates. A, Competing risks after TAVR for patients assigned to the transfemoral (TF) stratum, that is, the TF-TAVR subgroup. B, Competing risks after TAVR for patients assigned to the transapical (TA) stratum, that is, the TA-TAVR subgroup. $\mathrm{C}$, Competing risks after AVR for patients assigned to the transfemoral (TF) stratum. D, Competing risks after AVR for patients assigned to the transapical (TA) stratum. Note that follow-up has been truncated at 1.9 years when less than $10 \%$ of patients in this subgroup remained at risk. 


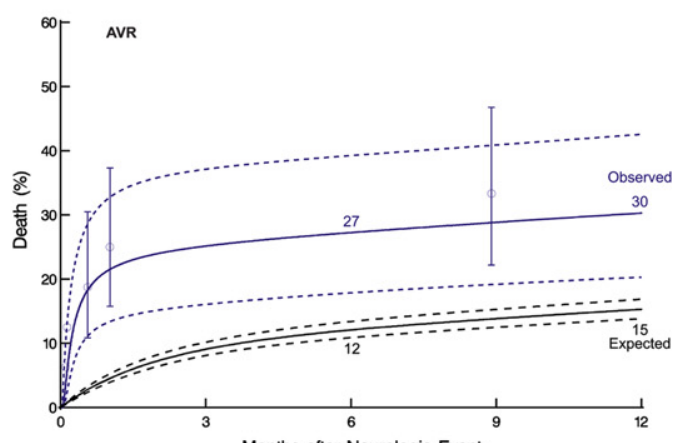

Atrisk 16 Months after Neurologic Event
13

A

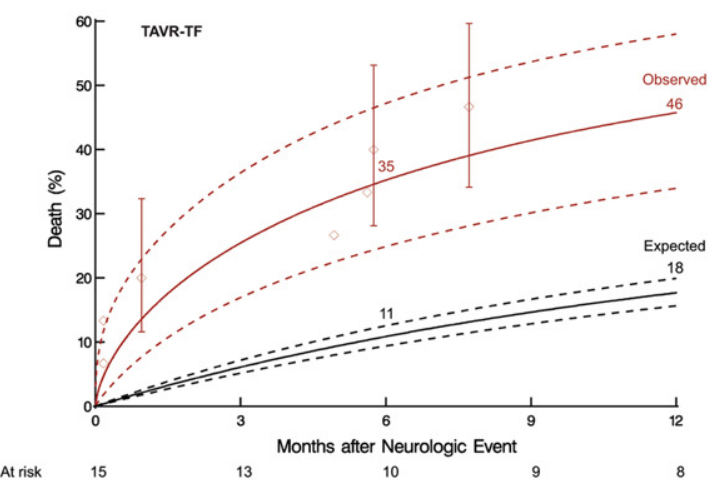

C

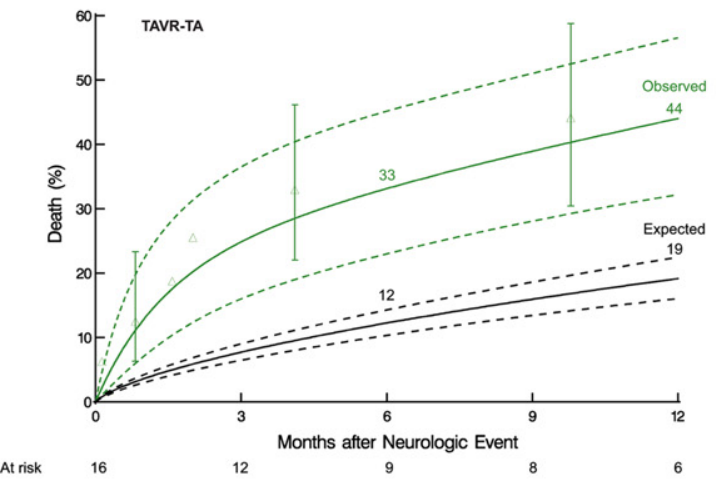

E

FIGURE E5. Mortality after a neurologic event following transcatheter aortic valve replacement (TAVR) and surgical aortic valve replacement (AVR). A, Mortality after a neurologic event in the AVR treatment arm over the first postoperative year. Each symbol represents a death; vertical bars are confidence limits equivalent to \pm 1 standard error, and solid lines enclosed within dashed confidence bands indicating \pm 1 standard error represent parametric estimates. The blue curve is observed mortality and the black curve represents an estimate of mortality had a neurologic event not occurred. B, Early hazard function for death out to 3 months after a neurologic event for the AVR treatment arm. The blue curve is observed hazard and the black curve is hazard function for mortality as if a neurologic event had not occurred. C, Mortality after a neurologic event in the TF-TAVR treatment arm over the first postoperative year. The depiction is as in Figure E5, A. The red curve is observed mortality and the black curve enclosed in a dashed confidence band equivalent to 1 standard error represents an estimate of mortality had a neurologic event not occurred. D, Early hazard function for death out to 3 months for mortality after a neurologic event following TF-TAVR. The red curve is observed hazard and the black curve is hazard function for mortality as if a neurologic event had not occurred. E, Mortality after a neurologic event in the TA-TAVR treatment arm over the first postoperative year. The depiction is as in Figure E5, A. The green curve is observed mortality and the black curve enclosed in a dashed confidence band equivalent to 1 standard error represents an estimate of mortality had a neurologic event not occurred. F, Early hazard function for death out to 3 months after a neurologic event in the TA-TAVR treatment arm. The green curve is observed hazard and the black curve is hazard function for mortality as if a neurologic event had not occurred. 
TABLE E1. Baseline characteristics of "as treated" (AT) patients assigned to TF or TA stratum

\begin{tabular}{|c|c|c|c|}
\hline Variable & TA $(\mathbf{n}=196)$ & TF $(n=461)$ & $P$ value \\
\hline Age (y) & $83.2 \pm 6.3$ & $84.3 \pm 6.7$ & .04 \\
\hline Female gender & $88 / 196(44.9 \%)$ & $192 / 461(41.6 \%)$ & .49 \\
\hline STS risk score & $11.9 \pm 3.6$ & $11.7 \pm 3.3$ & .53 \\
\hline Logistic EuroSCORE & $29.9 \pm 15.5$ & $29.0 \pm 16.0$ & .54 \\
\hline NYHA class III or IV & $184 / 196(93.9 \%)$ & $437 / 461(94.8 \%)$ & .71 \\
\hline Body surface area $\left(\mathrm{m}^{2}\right)$ & $1.8 \pm 0.3$ & $1.8 \pm 0.2$ & .97 \\
\hline Carotid endarterectomy/stent & $40 / 192(20.8 \%)$ & $36 / 452(8.0 \%)$ & $<.01$ \\
\hline Peripheral arterial bypass graft & $11 / 191(5.8 \%)$ & $6 / 455(1.3 \%)$ & $<.01$ \\
\hline Other arterial stent or PTA & $21 / 191(11.0 \%)$ & $15 / 455(3.3 \%)$ & $<.01$ \\
\hline Stroke or TIA within last $6-12$ mo & $7 / 191(3.7 \%)$ & $12 / 458(2.6 \%)$ & .45 \\
\hline Previous CABG & $102 / 194(52.6 \%)$ & $182 / 457(39.8 \%)$ & $<.01$ \\
\hline No. of previous CABGs & $82 / 102(80.4 \%)$ & $155 / 184(84.2 \%)$ & .71 \\
\hline Coronary artery disease & $153 / 194(78.9 \%)$ & $346 / 459(75.4 \%)$ & .37 \\
\hline Previous $\mathrm{Q}$ wave or non-Q wave MI & $62 / 192(32.3 \%)$ & $120 / 454(26.4 \%)$ & .15 \\
\hline Previous PCI & $72 / 187(38.5 \%)$ & $144 / 455(31.6 \%)$ & .10 \\
\hline Cerebrovascular disease & $66 / 177(37.3 \%)$ & $108 / 428(25.2 \%)$ & $<.01$ \\
\hline Peripheral vascular disease & $120 / 192(62.5 \%)$ & $159 / 455(34.9 \%)$ & $<.01$ \\
\hline COPD—any & $87 / 136(64.0 \%)$ & $200 / 313(63.9 \%)$ & 1.00 \\
\hline Oxygen-dependent pulmonary disease & $19 / 138(13.8 \%)$ & $53 / 319(16.6 \%)$ & .49 \\
\hline Previous pacemaker implant & $38 / 194(19.6 \%)$ & $101 / 458(22.1 \%)$ & .53 \\
\hline Pulmonary hypertension & $93 / 194(47.9 \%)$ & $229 / 420(54.5 \%)$ & .14 \\
\hline Creatinine & $1.5 \pm 2.0$ & $1.6 \pm 3.2$ & .45 \\
\hline Atrial fibrillation (ECG Core Lab) & $46 / 168(27.4 \%)$ & $99 / 408(24.3 \%)$ & .46 \\
\hline CHADS2 stroke risk score & $2.5 \pm 0.7$ & $2.4 \pm 0.7$ & .36 \\
\hline Deleterious effects of chest-wall irradiation & $2 / 196(1.0 \%)$ & $3 / 461(0.7 \%)$ & .64 \\
\hline Liver disease & $2 / 192(1.0 \%)$ & $13 / 458(2.8 \%)$ & .25 \\
\hline Coronary artery stenosis $>50 \%$ & $124 / 194(63.9 \%)$ & $263 / 463(56.8 \%)$ & .07 \\
\hline Aortic valve annulus diameter $(\mathrm{cm})$ & $2.0 \pm 0.2$ & $2.0 \pm 0.2$ & .99 \\
\hline Aortic valve mean gradient (mmHg) & $41.1 \pm 13.4$ & $43.9 \pm 14.8$ & .03 \\
\hline Aortic valve area $\left(\mathrm{cm}^{2}\right)$ & $0.7 \pm 0.2$ & $0.6 \pm 0.2$ & .32 \\
\hline Aortic valve area index $\left(\mathrm{cm}^{2} / \mathrm{m}^{2}\right)$ & $0.4 \pm 0.1$ & $0.4 \pm 0.1$ & .47 \\
\hline LV ejection fraction $(\%)$ & $53.5 \pm 11.5$ & $52.9 \pm 13.6$ & .56 \\
\hline
\end{tabular}

All continuous variables expressed as mean \pm 1 standard deviation. $T F$, Transfemoral; TA, transapical; STS, Society of Thoracic Surgeons; NYHA, New York Heat association; $P T A$, percutaneous transluminal angioplasty; $T I A$, transient ischemic attack; $C A B G$, coronary artery bypass grafting; $M I$, myocardial infarction; $P C I$, percutaneous coronary intervention; COPD, chronic obstructive pulmonary disease; ECG, electrocardiogram; CHADS2, Congestive heart failure, Hypertension, Age, Diabetes mellitus, prior Stroke or transient ischemic attack; $L V$, left ventricular. 
TABLE E2. Parsimonious logistic regression model to identify patient-related and disease-related factors that were associated with a higher likelihood of being deemed to be a "non-TF candidate" for TAVR

\begin{tabular}{lccc}
\hline \multicolumn{1}{c}{ Risk factor } & Coefficient \pm SD & $\boldsymbol{P}$ value & R $(\%)$ \\
\hline $\begin{array}{l}\text { History of peripheral vascular } \\
\quad \text { disease }\end{array}$ & $0.84 \pm 0.19$ & $<.0001$ & 98 \\
Carotid endarterectomy/stent & $0.86 \pm 0.28$ & .002 & 78 \\
Female & $0.72 \pm 0.21$ & .0008 & 82 \\
History of CABG & $0.51 \pm 0.21$ & .02 & 61 \\
Lower AV mean gradient & $-0.020 \pm 0.0072$ & .006 & 58 \\
Recent date of surgery/implant & $1.4 \pm 0.22$ & $<.0001$ & 100 \\
\hline
\end{tabular}

$R(\%)$, Bagging reliability; $C A B G$, coronary artery bypass grafting; $A V$, aortic valve; $S D$, standard deviation. C-statistic $=0.75$. Note: The "recent date of surgery/implant" term is explained by the fact that the transapical (TA) limb of the transcatheter aortic valve replacement (TAVR) treatment arm did not start until 1 year after the transfemoral $(T F)$ limb. Also, centers were not permitted to commence TA-TAVR cases until after they had gained a substantial experience with TF-TAVR cases.

TABLE E3. Baseline characteristics of patient "as treated" (AT) population according to randomized treatment arm (TAVR vs AVR)

\begin{tabular}{|c|c|c|c|}
\hline Variable & $\operatorname{AVR}(\mathbf{n}=313)$ & TAVR $(n=344)$ & $P$ value \\
\hline Age (y) & $84.4 \pm 6.3$ & $83.6 \pm 6.8$ & .12 \\
\hline Female gender & $134 / 313(42.8 \%)$ & $146 / 344(42.4 \%)$ & .94 \\
\hline STS risk score & $11.7 \pm 3.4$ & $11.8 \pm 3.3$ & .65 \\
\hline Logistic EuroSCORE & $29.2 \pm 15.2$ & $29.4 \pm 16.5$ & .90 \\
\hline NYHA class III or IV & $297 / 313(94.9 \%)$ & $324 / 344(94.2 \%)$ & .73 \\
\hline Body surface area $\left(\mathrm{m}^{2}\right)$ & $1.8 \pm 0.2$ & $1.8 \pm 0.3$ & .89 \\
\hline Carotid endarterectomy/stent & $29 / 307(9.4 \%)$ & $47 / 337(13.9 \%)$ & .09 \\
\hline Peripheral arterial bypass graft & $7 / 306(2.3 \%)$ & $10 / 340(2.9 \%)$ & .63 \\
\hline Other arterial stent or PTA & $15 / 307(4.9 \%)$ & $21 / 339(6.2 \%)$ & .50 \\
\hline Stroke or TIA within 6-12 mo & $9 / 309(2.9 \%)$ & $10 / 340(2.9 \%)$ & 1.00 \\
\hline Previous CABG & $139 / 310(44.8 \%)$ & $145 / 341(42.5 \%)$ & .58 \\
\hline No. of previous CABGs & $112 / 139(80.6 \%)$ & $125 / 147(85.0 \%)$ & .46 \\
\hline Coronary artery disease & $241 / 310(77.7 \%)$ & $258 / 343(75.2 \%)$ & .46 \\
\hline Previous Q wave or non-Q wave MI & $90 / 307(29.3 \%)$ & $92 / 339(27.1 \%)$ & .54 \\
\hline Previous PCI & $101 / 305(33.1 \%)$ & $115 / 337(34.1 \%)$ & .80 \\
\hline Cerebrovascular disease & $79 / 285(27.7 \%)$ & $95 / 320(29.7 \%)$ & .65 \\
\hline Peripheral vascular disease & $132 / 307(43.0 \%)$ & $147 / 340(43.2 \%)$ & 1.00 \\
\hline COPD & $138 / 214(64.5 \%)$ & $149 / 235(63.4 \%)$ & .84 \\
\hline Oxygen-dependent pulmonary disease & $34 / 220(15.5 \%)$ & $38 / 237(16.0 \%)$ & .90 \\
\hline Previous pacemaker implant & $70 / 311(22.5 \%)$ & $69 / 341(20.2 \%)$ & .50 \\
\hline Pulmonary hypertension & $150 / 293(51.2 \%)$ & $172 / 321(53.6 \%)$ & .57 \\
\hline Creatinine & $1.6 \pm 3.3$ & $1.5 \pm 2.5$ & .71 \\
\hline Atrial fibrillation (ECG Core Lab) & $66 / 269(24.5 \%)$ & $79 / 307(25.7 \%)$ & .77 \\
\hline CHADS2 stroke risk score & $2.5 \pm 0.7$ & $2.4 \pm 0.7$ & .31 \\
\hline Deleterious effects of chest wall irradiation & $2 / 313(0.6 \%)$ & $3 / 344(0.9 \%)$ & 1.00 \\
\hline Liver disease & $8 / 310(2.6 \%)$ & $7 / 340(2.1 \%)$ & .80 \\
\hline Coronary artery stenosis $>50 \%$ & $184 / 313(58.8 \%)$ & $203 / 344(59.0 \%)$ & 1.00 \\
\hline Aortic valve annulus diameter $(\mathrm{cm})$ & $2.0 \pm 0.2$ & $2.0 \pm 0.2$ & .93 \\
\hline Aortic valve mean gradient $(\mathrm{mm} \mathrm{Hg}$ ) & $43.5 \pm 14.3$ & $42.7 \pm 14.5$ & .51 \\
\hline Aortic valve area $\left(\mathrm{cm}^{2}\right)$ & $0.6 \pm 0.2$ & $0.7 \pm 0.2$ & .32 \\
\hline Aortic valve area index $\left(\mathrm{cm}^{2} / \mathrm{m}^{2}\right)$ & $0.4 \pm 0.1$ & $0.4 \pm 0.1$ & .31 \\
\hline LV ejection fraction $(\%)$ & $53.6 \pm 12.5$ & $52.6 \pm 13.5$ & .35 \\
\hline
\end{tabular}

All continuous variables expressed as mean \pm 1 standard deviation. TF, Transfemoral; TA, transapical; STS, Society of Thoracic Surgeons; NYHA, New York Heat association; $P T A$, percutaneous transluminal angioplasty; $T I A$, transient ischemic attack; $C A B G$, coronary artery bypass grafting; $M I$, myocardial infarction; $P C I$, percutaneous coronary intervention; $C O P D$, chronic obstructive pulmonary disease; $E C G$, electrocardiogram; CHADS2, Congestive heart failure, Hypertension, Age, Diabetes mellitus, prior Stroke or transient ischemic attack; $L V$, left ventricular. 
TABLE E4. Selected baseline characteristics for the 4 subgroups of AT patients categorized by assigned stratum (TF vs TA) and randomized treatment arm (TAVR vs AVR)

\begin{tabular}{|c|c|c|c|c|}
\hline \multirow[b]{2}{*}{ Variable } & \multicolumn{2}{|c|}{ TA stratum } & \multicolumn{2}{|c|}{ TF stratum } \\
\hline & $\operatorname{AVR}(\mathbf{n}=92)$ & TA-TAVR $(n=104)$ & $\operatorname{AVR}(\mathbf{n}=\mathbf{2 2 1})$ & TF-TAVR $(n=240)$ \\
\hline Age (y) $( \pm 1 \mathrm{SD})$ & $83.4 \pm 5.5$ & $82.9 \pm 7.0$ & $84.8 \pm 6.6$ & $83.9 \pm 6.8$ \\
\hline STS risk score & $12.1 \pm 3.5$ & $11.7 \pm 3.6$ & $11.5 \pm 3.3$ & $11.9 \pm 3.2$ \\
\hline Logistic EuroSCORE & $29.9 \pm 15.1$ & $29.9 \pm 16.0$ & $28.9 \pm 15.2$ & $29.1 \pm 16.7$ \\
\hline NYHA class III or IV & $96 \%$ & $92 \%$ & $95 \%$ & $95 \%$ \\
\hline Carotid endarterectomy/stent & $17 \%$ & $24 \%$ & $6 \%$ & $10 \%$ \\
\hline Stroke or TIA within last $6-12 \mathrm{mo}$ & $7 \%$ & $1 \%$ & $1 \%$ & $4 \%$ \\
\hline Previous CABG & $56 \%$ & $50 \%$ & $40 \%$ & $40 \%$ \\
\hline Coronary artery disease & $84 \%$ & $75 \%$ & $75 \%$ & $75 \%$ \\
\hline Previous $\mathrm{Q}$ wave or non-Q wave $\mathrm{MI}$ & $38 \%$ & $28 \%$ & $26 \%$ & $27 \%$ \\
\hline Cerebrovascular disease & $31 \%$ & $43 \%$ & $26 \%$ & $24 \%$ \\
\hline Peripheral vascular disease & $62 \%$ & $63 \%$ & $35 \%$ & $35 \%$ \\
\hline COPD & $64 \%$ & $64 \%$ & $65 \%$ & $63 \%$ \\
\hline Pulmonary hypertension & $42 \%$ & $53 \%$ & $55 \%$ & $54 \%$ \\
\hline Atrial fibrillation & $21 \%$ & $32 \%$ & $26 \%$ & $23 \%$ \\
\hline Mean aortic valve gradient $(\mathrm{mm} \mathrm{Hg})$ & $40.5 \pm 12.9$ & $41.7 \pm 13.9$ & $44.7 \pm 14.8$ & $43.1 \pm 14.8$ \\
\hline Aortic valve area $\left(\mathrm{cm}^{2}\right)$ & $0.7 \pm 0.2$ & $0.7 \pm 0.2$ & $0.6 \pm 0.2$ & $0.7 \pm 0.2$ \\
\hline Aortic valve area index $\left(\mathrm{cm}^{2} / \mathrm{m}^{2}\right)$ & $0.4 \pm 0.1$ & $0.4 \pm 0.1$ & $0.3 \pm 0.1$ & $0.4 \pm 0.1$ \\
\hline LV ejection fraction $(\%)$ & $53.5 \pm 10.9$ & $53.6 \pm 12.2$ & $53.6 \pm 13.1$ & $52.2 \pm 14.0$ \\
\hline
\end{tabular}

All continuous variables expressed as mean \pm 1 standard deviation. TF, Transfemoral; TA, transapical; $S D$, standard deviation; STS, Society of Thoracic Surgeons; NYHA, New York Heat association; $T I A$, transient ischemic attack; $C A B G$, coronary artery bypass grafting; $M I$, myocardial infarction; $C O P D$, chronic obstructive pulmonary disease; $L V$, left ventricular.

TABLE E5. Procedural details of patient population (AT) according to randomized treatment arm (TAVR vs AVR)

\begin{tabular}{|c|c|c|c|}
\hline & TAVR $(n=344)$ & & $\operatorname{AVR}(\mathbf{n}=313)$ \\
\hline Days to implant & $10.8 \pm 13.8$ & Days to implant & $15.6 \pm 19.7$ \\
\hline Anesthesia duration (min) & $236.3 \pm 96.4$ & Anesthesia duration (min) & $330.3 \pm 104.4$ \\
\hline Total procedure time $(\mathrm{min})$ & $133.1 \pm 88.6$ & Total procedure time $(\mathrm{min})$ & $230.0 \pm 45.5$ \\
\hline Fluoroscopy time (min) & $31.3 \pm 75.7$ & Total crossclamp time (min) & $73.5 \pm 28.7$ \\
\hline Aborted procedure, no. $(\%)$ & $7(2.0 \%)$ & Pump time (min) & $104.9 \pm 41.4$ \\
\hline Converted procedure, no. $(\%)$ & $9(2.6 \%)$ & Aborted procedure, no. $(\%)$ & $0(0 \%)$ \\
\hline Due to valve embolization & 5 & Converted procedure, no. $(\%)$ & $1(0.3 \%)$ \\
\hline Due to annulus size on TEE & 3 & Due to extremely calcified aorta & 1 \\
\hline Due to large sigmoid septum & 1 & & \\
\hline Reoperation for bleeding, no. $(\%)$ & $2(0.6 \%)$ & Reoperation for bleeding, no. $(\%)$ & $12(3.4 \%)$ \\
\hline Intraprocedural death, no. $(\%)$ & $3(0.9 \%)$ & Intraprocedural death, no. $(\%)$ & $1(0.3 \%)$ \\
\hline Vascular access infection, no. $(\%)$ & $7(2.0 \%)$ & Sternal wound infection, no. $(\%)$ & $7(2.0 \%)$ \\
\hline $\begin{array}{l}\text { Valve embolization (not reported as } \\
\text { conversion or valve-in-valve), no. ( } \%)\end{array}$ & $2(0.6 \%)$ & & \\
\hline Multiple valve ( $\geq 2$ implanted), no. (\%) & $7(2.0 \%)$ & & \\
\hline Due to valve embolization & 2 & & \\
\hline Due to residual aortic regurgitation & 5 & & \\
\hline Coronary obstruction, no. $(\%)$ & $0(0.0 \%)$ & & \\
\hline Aortic perforation-no. (\%) & $0(0.0 \%)$ & Aortic perforation, no. $(\%)$ & $1(0.3 \%)$ \\
\hline Aortic dissection, no. (\%) & $3(0.9 \%)$ & Aortic dissection, no. (\%) & $2(0.6 \%)$ \\
\hline Average ICU stay (d) & 5.9 median $=3.0(\mathrm{IQR} 2,6)$ & Average ICU stay (d) & 8.4 median $=5.0(\mathrm{IQR} 3,8)$ \\
\hline Average index hospital stay (d) & 11.7 median $=8($ IQR 5,14$)$ & Average index hospital stay (d) & 16.7 median $=12(\mathrm{IQR} 8,20)$ \\
\hline
\end{tabular}


TABLE E6. Preprocedural variables considered in analyses

\section{Demographics}

Age at screening

Gender

Clinical Status

NYHA class

Logistic EuroSCORE

STS risk score

CHADS2 stroke risk score

Aortic valve pathology

Aortic valve annular diameter

Aortic valve area

Aortic valve area index

Aortic valve mean gradient

Endocarditis

Cardiac comorbidity

Moderate or severe mitral regurgitation

Coronary artery stenosis $>50 \%$ ?

Coronary artery disease

No, of previous CABGs

Prior CABG

Prior PCI

Previous $\mathrm{Q}$ wave or non-Q wave MI

LV ejection fraction

Atrial fibrillation (ECG Core Lab)

History of atrial fibrillation or other arrhythmias

Pacemaker implant

Cardiovascular comorbidity

Cerebrovascular disease

Stroke or TIA within last 6-12 mo

Carotid endarterectomy/stent

No. of prior aortic valvuloplasties

Porcelain aorta

Aortic dissection

Peripheral vascular disease

Peripheral bypass graft

Other arterial stent or PTA

Pulmonary hypertension

Deleterious effects of chest wall irradiation

Transapical vs transfemoral assigned stratum

Noncardiac comorbidity

Liver disease

Creatinine

COPD

Oxygen-dependent COPD

Treatment variables

AT trial arm (computed)

AT implant approach

Implant procedure date

NYHA, New York Heat Association; STS, Society of Thoracic surgeons; CHADS2, Congestive heart failure, Hypertension, Age, Diabetes mellitus, prior Stroke or transient ischemic attack; $C A B G$, coronary artery bypass grafting; $P C I$, percutaneous coronary intervention; $M I$, myocardial infarction; $L V$, left ventricular; $E C G$, electrocardiogram; TIA, transient ischemic attack; PTA, percutaneous transluminal angioplasty; $C O P D$, chronic obstructive pulmonary disease.
TABLE E7. Procedural variables considered in analyses

\author{
TAVR-specific variables \\ Implant approach (as treated) \\ Fluoroscopy total time (min) \\ Aortic perforation \\ Second THV valve implanted \\ Device embolization \\ Conversion to open cardiac surgery \\ Surgical AVR-specific variables \\ Total aortic crossclamp time \\ Anesthesia duration \\ Pump time \\ General procedure variables \\ Valve size \\ Derived device size
}

TAVR, Transcatheter aortic valve replacement; $T H V$, transcatheter heart valve; $A V R$, aortic valve replacement. 